\title{
Warum schrumpft die migrantische Mittelschicht in der Stadt? Zur Dynamik der Schichtung am Beispiel Wiens zwischen 2003 und 2013
}

\author{
Bernhard Riederer · Lena Seewann - Roland Verwiebe
}

Online publiziert: 10. Dezember 2018

(C) Der/die Autor(en) 2018

Zusammenfassung Die internationale Stadtforschung diskutiert aktuell verstärkt in den letzten Jahrzehnten stattfindende Veränderungen in der Sozialstruktur westlicher Gesellschaften, da Städte und urbane Ballungsräume hiervon besonders betroffen sind. Obwohl die Migration in theoretischen Debattenbeiträgen von Beginn an als wesentlich für die Re-Strukturierung der urbanen Sozialstruktur angesehen wurde, existiert ein Mangel an empirischen Studien, die deren Rolle explizit und detailliert untersuchen. Der vorliegende Beitrag analysiert daher am Beispiel Wiens die Bedeutung von Migration und deren Heterogenität für Veränderungen des städtischen Schichtgefüges. In logistischen Modellen und Dekompositionsanalysen prüfen wir systematisch die Relevanz migrationsspezifischer und arbeitsmarktrelevanter Charakteristika der Migrantenpopulation für die Schichtzugehörigkeit. In Wien ging der Anteil der Mittelschicht in den letzten zwei Dekaden gerade unter Personen mit Migrationshintergrund dramatisch zurück. Unsere Ergebnisse zeigen unter anderem,

Online-Anhang: http://www.kzfss.uni-koeln.de/sites/kzfss/pdf/verwiebe_et_al.pdf

Aus Gründen der besseren Lesbarkeit wird auf die gleichzeitige Verwendung männlicher und weiblicher Sprachformen verzichtet. Sämtliche Personenbezeichnungen gelten gleichermaßen für beiderlei Geschlecht.

\footnotetext{
B. Riederer $\cdot$ L. Seewann $\cdot$ R. Verwiebe $(\square)$

Institut für Soziologie, Universität Wien

Rooseveltplatz 2, 1090 Wien, Österreich

E-Mail: roland.verwiebe@univie.ac.at

B. Riederer

E-Mail: bernhard.riederer@univie.ac.at

L. Seewann

E-Mail: lena.seewann@univie.ac.at

B. Riederer

Institut für Demographie, Österreichische Akademie der Wissenschaften

Welthandelsplatz 2, 1020 Wien, Österreich
} 
dass das Schrumpfen der Mittelschicht sogar noch deutlicher ausgefallen wäre, wenn die Migranten aktuell dieselben Charakteristika (z. B. bzgl. Herkunft und Bildungsgrad) wie noch vor einigen Jahren aufweisen würden.

Schlüsselwörter Schrumpfende Mittelschicht $\cdot$ Sozialstruktur $\cdot$ Migration $\cdot$ Soziale Ungleichheit · Wien

\title{
Why is the Migrant Middle Class Shrinking in Urban Areas? The Example of the Changing Social Stratification in Vienna Between 2003 and 2013
}

\begin{abstract}
The changing social stratification within western societies became especially evident in cities in the last decades. Therefore, stratification dynamics are increasingly discussed in international urban research. Although migration is considered essential for the restructuring of urban social stratification in theoretical debates, there is a lack of empirical studies addressing the explicit role migration plays. The present article analyses the relevance of migration patterns and the heterogeneity of migrant populations taking Vienna as an example. Logistic models and decomposition analysis systematically examine effects of migration specific and labour market specific characteristics of the migrant population for their social position and ongoing changes within the urban class structure. The share of middle class households has declined significantly among migrants in Vienna within the last two decades. Our results indicate, among other things, that the shrinking of the migrant middle class would have been even more pronounced, if migrants still possessed the characteristics (e.g. country of origin, education) that were typical for this group a few years earlier.
\end{abstract}

Keywords Shrinking of middle class $\cdot$ Social structure $\cdot$ Migration $\cdot$ Social inequality · Vienna

\section{Einleitung}

Die sozialwissenschaftliche Forschung hat in den letzten Jahren vielfach herausgearbeitet, dass das Schichtungsgefüge fortgeschrittener westlicher Gesellschaften in einem Wandel begriffen ist, der zu steigender Ungleichheit führt (z. B. Alderson et al. 2005; Atkinson und Brandolini 2011; Piketty 2014). Als ein wesentlicher Grund für diesen Prozess gelten die wirtschaftlichen Prozesse der Tertiarisierung und Globalisierung, welche zu einem Verlust gut bezahlter Industriejobs und zu einem Anstieg der Niedriglohnbeschäftigung geführt haben (Dallinger 2013; Lucifora et al. 2005). Der Wandel von der Industrie- zur Dienstleistungsgesellschaft erhöht jedoch auch die Nachfrage nach hochqualifizierten Fachkräften (Acemoglu 2002; Autor et al. 2006; Buera und Kaboski 2012; Esping-Andersen 2007). Beide Entwicklungen führen dazu, dass die Mittelschicht in vielen westlichen Gesellschaften (stark) geschrumpft ist (u.a. Böhnke 2010; Foster und Wolfson 2010; Grabka und 
Frick 2008; Groh-Samberg et al. 2014; Massari et al. 2009; Mau 2012; Whelan et al. 2017).

Einen besonderen Stellenwert haben diese Entwicklungen in den Debatten der Stadtforschung eingenommen (u.a. Boterman et al. 2018; Butler 2003; Hamnett 2015; May et al. 2007; Savage et al. 2013). Die Polarisierung des Arbeitsmarktes (Sassen 2016; Tai 2006) und daraus resultierende Einkommensunterschiede (Burgers und Musterd 2002; Goebel et al. 2012; Hamnett 1994) sind in den Städten am deutlichsten ausgeprägt. Die Folgen sind wachsende Ungleichheit und eine zunehmende soziale Polarisierung städtischer Gesellschaften (Burgers und Musterd 2002; Goebel et al. 2012; Musterd et al. 2017). Das Ausmaß dieser Polarisierung ist jedoch umstritten. In zahlreichen Städten ist auch das Entstehen neuer Mittelschichten feststellbar (Hamnett 2015, S. 240). Zudem haben Städte die Möglichkeit, ökonomische Prozesse durch lokale Politik zu beeinflussen und der wachsenden Ungleichheit entgegen zu wirken (Le Galès 2002; Musterd und Ostendorf 2013).

Mit den vorliegenden Ausarbeitungen wollen wir zu dieser Diskussion beitragen, indem wir die Entwicklung der migrantischen Mittelschicht im urbanen Raum thematisieren. Einige Studien haben bereits explizit auf den Bedarf einer systematischen Untersuchung des Wandels der städtischen Sozialstruktur unter spezifischer Berücksichtigung des Faktors Migration hingewiesen (z.B. Bailey et al. 2017; Hamnett 2015; May et al. 2007; Watt 2008). Diese Studien zeigen, dass internationale Migration entscheidend zur Polarisierung der sozialen Schichtung im urbanen Raum beiträgt (McDowell et al. 2009; Tai 2006). ${ }^{1}$ Wir wollen diesen Zusammenhang am Beispiel Wiens mithilfe von umfassenden statistischen Analysen detailliert untersuchen.

Wien ist für eine solche Untersuchung aus verschiedenen Gründen ein interessanter Fall. Zum einen hat die Stadt durch neue Formen der Zuwanderung in den letzten zwei Dekaden einen starken Bevölkerungszuwachs erlebt. Rund 29\% der in Wien lebenden Menschen sind keine österreichischen Staatsbürger, 1995 lag dieser Bevölkerungsanteil noch bei weniger als $12 \%$. Diese demografische Entwicklung korrespondiert mit einem Strukturwandel von der Industrie- zur Dienstleistungsgesellschaft, der für den Wiener Arbeitsmarkt massive Folgen hatte (Fassmann et al. 2009, S. 31; Stadt Wien 2016a). Aktuell sind nur noch 7,5\% der Erwerbstätigen in der Industrie tätig, damit gingen seit 1995 etwa 83.000 Industriejobs verloren, ${ }^{2}$ gleichzeitig hat die Einkommensungleichheit in Wien stetig zugenommen (Aschauer et al. 2012, S. 62; Görgl et al. 2011). Wien spiegelt auch den internationalen Trend, dass Migranten von negativen Folgen ökonomischer Krisen besonders betroffen sind (z.B. Leschke und Galgögczi 2015; Lohmann 2010), wider: $40 \%$ der Arbeitslosen

\footnotetext{
1 Tai (2006, S. 1753) argumentiert zum Beispiel so: ,,[S]ocial polarization in Singapore, Hong Kong and Taipei occurs primarily in the external migrant labor market rather than in the internal social structure [of the native population]." Auch Watt (2008, S. 209) verweist in seiner Studie auf die besondere Bedeutung von Migration für das Schichtungsgefüge (am Beispiel Londons): „,T]hese [lower class] fractions include a new migrant 'reserve army of labour' doing many of the city's socially invisible, often 'dirty jobs' (cleaning, caring, etc.); alongside white and established black and Asian groups who intermit over time between routine employment (manual and non-manual) and various forms of non-employment."

2 Die Zahlen zur Branchenstruktur und zur Zuwanderung beruhen auf dem Österreichischen Mikrozensus der Jahre 1995 und 2017 (eigene Berechnungen).
} 
in Wien besitzen eine ausländische Staatsbürgerschaft (AMS 2016). Der Anteil der Migranten nahm zwar in den letzten 20 Jahren in hoch qualifizierten Berufen und Berufen mit Managementaufgaben überproportional stark zu, doch Migranten gehen auch überdurchschnittlich häufig Hilfsarbeitstätigkeiten nach (Fritsch et al. 2018). ${ }^{3}$ Damit ist die wachsende Zuwanderung in Wien ein substanzieller Faktor für die Restrukturierung des Schichtungsgefüges der Stadt.

In unserer Untersuchung wollen wir erörtern, welche Folgen die Veränderung der Migrationsströme für das städtische Schichtgefüge hat. Damit verknüpft beschäftigen wir uns mit der Frage, welche spezifische Relevanz Faktoren wie regionale Herkunft, (Aus)Bildung oder Sprachkenntnisse, aber auch verschiedene Facetten der Arbeitsmarktintegration für das Erreichen unterschiedlicher sozialer Positionen für Migranten besitzen. Der dazu herangezogene Datensatz zur Lebenssituation der Wiener Stadtbevölkerung in den Jahren 2003 und 2013 zeichnet sich durch Repräsentativität für die Wohnbevölkerung der Stadt, eine für detaillierte Analysen ausreichend hohe Fallzahl bei Personen mit Migrationshintergrund ( $n>2000$ pro Welle) und die Erfassung einer Vielzahl an migrationsspezifischen Charakteristika (z.B. Herkunft, Sprachkenntnisse) aus.

\section{Literaturüberblick, Theorie und Hypothesen}

\subsection{Migration und der Wandel der Schichtung in der Stadt}

Die Frage der Umwälzung des Schichtungsgefüges im Zuge von Tertiarisierung, Globalisierung und demografischem Wandel ist mittlerweile zu einem der dominanten Themen der Stadtforschung geworden (Burgers und Musterd 2002; Butler 2003; Hamnett 2015; Savage et al. 2013). Die entsprechende Debatte geht auf die von Friedmann (1986) und Sassen (1991) vertretene „Polarisierungsthese“ zurück. Dieser These zufolge benötigt der städtische Arbeitsmarkt auf der einen Seite hochqualifizierte Fachkräfte und auf der anderen Seite ,,preiswerte“ Arbeitskräfte mit geringen Qualifikationen (Burgers und Musterd 2002; Goebel et al. 2012; Tai 2006), was zu wachsenden Einkommensunterschieden und einer Polarisierung der sozialen Schichtung führt. Jene Mittelschichten, die einst das Rückgrat der industriellen Konsumgesellschaft bildeten, verlieren demnach an Bedeutung: „What [the city] needs least are the traditional modest middle classes so central to the era when mass consumption was the dominant logic" (Sassen 2016, S. 98).

Friedmann (1986) und Sassen (1991) haben bereits früh die Bedeutung der Migration für die Re-Strukturierung und Polarisierung der urbanen Sozialstruktur hervorgehoben, was nur mit einer gewissen Verzögerung als Thema durch die empiri-

\footnotetext{
3 Zu guter Letzt verdeutlicht Wien die Bedeutung der Stadt für die Dynamik der sozialen Schichtung besonders gut. Denn während Wohlfahrtsstaat und Sozialpartnerschaft das Schichtgefüge und damit den Anteil der Mittelschicht in Österreich insgesamt in den letzten Jahrzehnten weitgehend stabil halten konnten - im internationalen Vergleich gilt Österreich als „Hort der Stabilität“ (Trampusch 2010; Verwiebe et al. 2014) -, nahm die Mittelschicht in Wien deutlich ab. Für detaillierte Ausführungen zu Wien im innerösterreichischen und internationalen Vergleich siehe den Online-Anhang (Abschn. A1).
} 
sche Forschung aufgegriffen wurde (Sanderson et al. 2015). ${ }^{4}$ Großstädte nehmen in der Regel unterschiedliche Gruppen von Migranten auf, die sowohl die Nachfrage nach hochqualifizierten als auch nach geringqualifizierten Arbeitskräften bedienen. Dieser Zuzug kann die Polarisierung der sozialen Schichtung im urbanen Raum beschleunigen (McDowell et al. 2009; Tai 2006). Zwar sind Migranten an europäischen Arbeitsmärkten traditionell benachteiligt (Kalter und Granato 2018; Leschke und Galgögczi 2015), doch versuchen Städte wie Wien mittlerweile gezielt, hochqualifizierte Fachkräfte anzuziehen (Ewers 2007; OECD 2008; Reiner et al. 2017; Tai 2006). Unterschiedliche Charakteristika verschiedener Migrantengruppen führen jedoch zu unterschiedlichem Erfolg am städtischen Arbeitsmarkt und zu Spaltungen innerhalb der migrantischen Arbeitskräftepopulation (Davidson und Wyly 2012; McDowell et al. 2009; OECD 2018). Für Europa kann festgehalten werden, dass hochqualifizierte Migranten überwiegend aus (anderen) EU-Mitgliedsstaaten sowie Nordamerika kommen, während geringqualifizierte Migranten häufig aus der Türkei, dem Mittleren Osten oder Nordafrika stammen (Kalter und Granato 2018; OECD 2018). Vor diesem Hintergrund wollen wir in unserem Beitrag die Rolle von Migrationsdynamiken für die soziale Schichtung Wiens in den Mittelpunkt unserer Analysen rücken. Zu diesem Zweck berücksichtigen wir neben arbeitsmarktbezogenen Entwicklungen auch migrationsspezifische Indikatoren (wie z.B. regionale Herkunft und den Bildungsstand unterschiedlicher Migrantengruppen).

Erste Befunde zu Wien führen die Relevanz von Migration für die Veränderung der Schichtung bereits deutlich vor Augen. Mehrere Zuwanderungswellen haben die Stadt im letzten Vierteljahrhundert stark geprägt (Abb. 1 verdeutlicht die damit einhergehende Veränderung der Wiener Bevölkerung): Während in den 1990er Jahren zunächst vor allem Flüchtlinge aus dem ehemaligen Jugoslawien und der Nachzug der Angehörigen früherer türkischer Migranten zu einem städtischen Bevölkerungswachstum führten, nahm ab der Jahrtausendwende der Anteil der EU-Bürger stark zu (Stadt Wien 2016b). Dabei handelte es sich primär um Menschen aus Ländern wie Deutschland, Polen oder Rumänien, die in der Regel wesentlich besser ausgebildet waren als frühere Generationen von Arbeitsmigranten. Auch die Zuwanderung nach Wien aus Drittstaaten nimmt seit 1990 kontinuierlich zu.

Parallel zur Veränderung der Zuwanderung und der migrantischen Population nahm der Anteil der Mittelschicht in Wien seit Anfang der 2000er Jahre je nach Datenquelle um fünf bis zehn Prozentpunkte ab (von rund 46 auf unter $40 \%$; vgl. Online Appendix A 1.3). Die Gruppe der Migranten war jedoch wesentlich stärker vom Schrumpfen der Mittelschicht betroffen als Einheimische: Während der Anteil der Mittelschicht, EU-SILC Daten zufolge, zwischen 2004 und 2015 unter den in

\footnotetext{
4 In der Stadtforschung gibt es einige Studien mit größeren Datensätzen, in denen Migration, neben anderen Faktoren, eine wichtige Erklärungsdimension ist (z. B. Butler et al. 2008; Cunningham und Savage 2017; Savage et al. 2013). Das Hauptaugenmerk der Stadtforschung liegt allerdings auf kleinräumlichen Analysen der Sozialstruktur (u. a. Franz 2015; Häußermann und Siebel 1993; Holm 2011; Huber 2013). In der Migrationsforschung gibt es Forschung zu ,highly skilled migration“, die aber überwiegend mit kleinen qualitativen Befragungen arbeitet (Beaverstock 2005; Koser und Salt 1997; Peixoto 2001; Ryan und Mulholland 2014; Salt 1992). In der Sozialstrukturforschung wird systematisch Schichtung und Migration erforscht, hier dominieren allerdings nationale oder international vergleichende Analysen (Burkhardt et al. 2013; Kogan 2011). Städte oder Metropolregionen sind nur selten Untersuchungsgegenstand.
} 


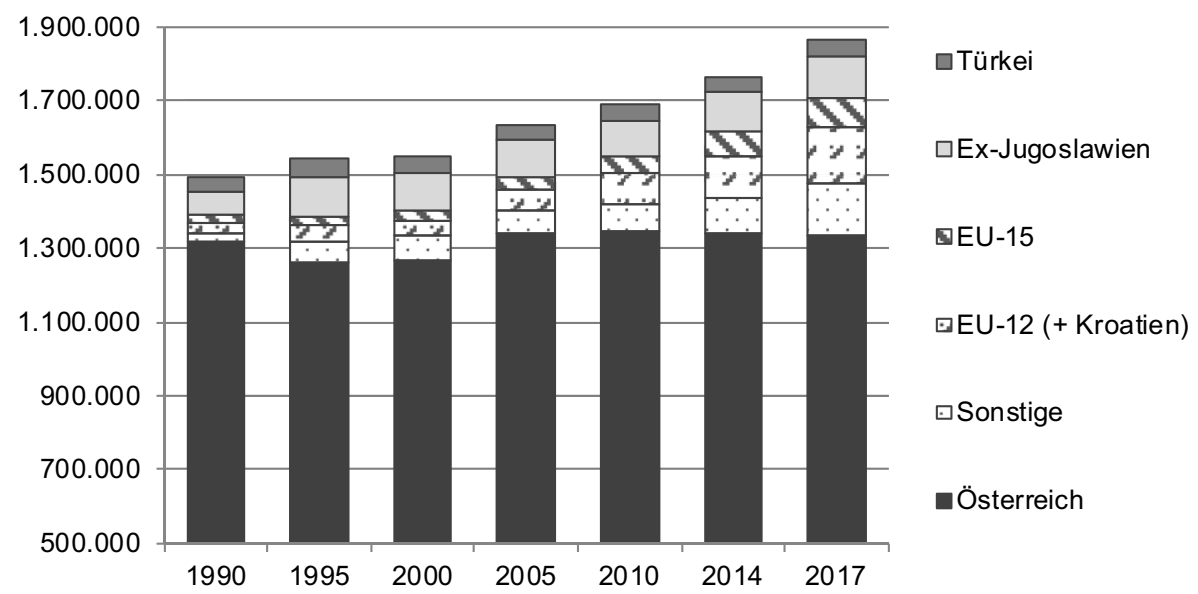

Abb. 1 Die Entwicklung der Wiener Bevölkerung nach Staatsbürgerschaft 1990-2017. Quelle: Verwiebe et al. (2015, S. 27; adaptierte Darstellung)

Österreich geborenen Personen nur von 47 auf $45 \%$ abnahm, fiel er bei im Ausland Geborenen um dreizehn Prozentpunkte (von 43 auf 36\%).

\subsection{Mögliche Ursachen für das Schrumpfen der migrantischen Mitte in Wien}

Um mögliche Ursachen für das Schrumpfen der migrantischen Mittelschicht erörtern zu können, soll in diesem Abschnitt zunächst dargelegt werden, warum bestimmte Faktoren für die Schichtung von Relevanz sind, bevor zeitliche Veränderungen thematisiert und mögliche Entwicklungen in Wien diskutiert werden. Theoretische Überlegungen werden zu zwei Klassen von Faktoren vorgebracht, die Veränderungen der Mittelschicht in der Gruppe der Personen mit Migrationshintergrund bewirken könnten: migrationsspezifische Faktoren auf der einen und arbeitsmarktbezogene Faktoren auf der anderen Seite. Damit fokussieren wir auf zwei zentrale Erklärungsebenen, die in der Migrationsforschung als relevant gelten (in den empirischen Analysen wird für weitere Einflussgrößen kontrolliert).

$\mathrm{Zu}$ den migrationsspezifischen Faktoren zählen zunächst Aufenthaltsdauer und rechtlicher Status (Staatsbürgerschaft). In der Literatur wird unter anderem angenommen, dass solche integrationsfördernde Faktoren einen positiven Einfluss auf die Schichtzugehörigkeit haben. Mit zunehmender Aufenthaltsdauer verstärkt sich die Akkulturation und Einbindung in soziale Netzwerke, wodurch Migranten bessere Positionen im Schichtungsgefüge einnehmen können (Kalter und Granato 2002; Siegert 2013, S. 177f.). Die Erlangung einer österreichischen Staatsbürgerschaft sorgt zudem für einen Wegfall rechtlicher Hürden. Sie erleichtert daher die Partizipation am Arbeitsmarkt (Zimmermann 2005) und gewährleistet darüber hinaus vollen Zugang zu wohlfahrtsstaatlichen Leistungen (Lohmann 2010, S. 25). Die zweite Generation, bereits in Österreich aufgewachsene Kinder von Migranten, besitzt im Vergleich zur ersten Generation häufiger die österreichische Staatsbürgerschaft, einen österreichischen Bildungsabschluss und ein regionales Netzwerk, die die Aufnah- 
me von Erwerbstätigkeit erleichtern. Sie sollte daher seltener von sozialem Abstieg betroffen sein als die Generation ihrer Eltern.

Relevant ist zudem das Herkunftsland von Migranten. Personen aus der Türkei sowie den Nachfolgestaaten des ehemaligen Jugoslawiens besitzen eine lange Tradition der Immigration nach Wien, die auf die Anwerbeabkommen aus den 1960er Jahren zurückzuführen ist (Bauer 2008; Gächter 2008). Im Gegensatz zu den Gastarbeitern der 1960er und 1970er Jahre sind diese Migrantengruppen inzwischen jedoch nicht mehr in vergleichbarem Ausmaß in gut bezahlten Industriejobs tätig (Giesecke und Verwiebe 2010; Wiedner und Giesecke 2017). Die Bürgerkriegsflüchtlinge der 1990er Jahre und durch Familienzusammenführungen eingewanderte Angehörige von Migranten aus der Türkei haben Schwierigkeiten, am Wiener Arbeitsmarkt Fuß zu fassen. Vor allem türkische Migranten leiden unter Bildungsnachteilen und stärkerer Diskriminierung (Dietze 2009; Verwiebe et al. 2016). Ihr Einkommen liegt rund ein Drittel unter dem Durchschnitt (vgl. Stadt Wien 2014; Statistik Austria 2015) und bestehende Benachteiligungen vergrößern sich in wirtschaftlichen Krisen oftmals. Ein Grund dafür ist auch, dass in diesen Gruppen die weibliche Erwerbstätigkeit, insbesondere bei Frauen aus der Türkei und Bosnien, sehr gering ist (Stadt Wien 2016a). Besonders problematisch ist die Arbeitsmarktlage zudem für Migranten aus Drittstaaten (ausgenommen USA, Kanada, Australien). Nur 56\% der Personen, die über einen Bildungsabschluss aus einem Drittstaat verfügen, sind erwerbstätig (Stadt Wien 2016a). Gerade die Anerkennung von Ausbildungstiteln stellt für diese Gruppe ein großes Problem dar. Migranten aus Drittstaaten üben daher häufig Tätigkeiten aus, für die sie (gemäß ihrer Ausbildung) überqualifiziert sind (Leschke und Galgögczi 2015; OECD 2018). Migranten aus EU-Staaten haben hingegen aufgrund des EU-Rechts einen besseren Zugang zum österreichischen Arbeitsmarkt als andere Migranten. Sie verfügen im Durchschnitt über höhere Qualifikationen als die einheimische Bevölkerung (Stadt Wien 2016b, 2016a; Verwiebe et al. 2015) und erzielen häufig auch höhere Einkommen (vgl. Stadt Wien 2014; Statistik Austria 2015). Die Charakteristika unterschiedlicher Migrationsgruppen lassen daher vermuten, dass in Wien überwiegend türkische und ex-jugoslawische Personen sowie Migranten aus Drittstaaten vom gesellschaftlichen Abstieg bedroht sind.

Wie viele Studien immer wieder bestätigen, ist auch das durch (Aus)Bildung erworbene Humankapital für den Status einer Person von entscheidender Bedeutung. ${ }^{5}$ Es sind in erster Linie gering qualifizierte Personen, die von sozialem Abstieg bedroht sind (Mohr 2007). Höhere Bildung geht mit höheren Gehältern, verminderten Risiken temporärer Beschäftigung sowie geringerer Arbeitslosigkeit und Armutsgefährdung einher (z.B. Abrassart 2013; Vandecasteele 2011). Für Migranten ist Bildung am Arbeitsmarkt vermutlich noch zentraler als für Menschen ohne Migrationshintergrund, um migrationsbedingte Nachteile aus kultureller Fremdheit oder fehlenden sozialen Beziehungen vor Ort kompensieren zu können. Da gesellschaft-

\footnotetext{
${ }^{5}$ Dafür werden in der Literatur mehrere Gründe angeführt: Allokationsmodelle betonen die Rolle des Bildungssystems für die Zuordnung zu gesellschaftlichen Positionen. Sozialisationstheorien schreiben das dem Erlernen verschiedenster Fertigkeiten im Laufe des Bildungsprozesses zu. Dem Humankapitalansatz zufolge erhöhen Investitionen in (Aus)Bildung tatsächlich die Produktivität. Die Signaltheorie versteht das Vorliegen von Bildungszertifikaten als Hinweis darauf, wie produktiv eine Person grundsätzlich sein dürfte (Barone und van de Werfhorst 2011).
} 
liche Positionen zu einem guten Teil ,vererbt“"werden, stellt Bildung für die Kinder der Einwanderer zudem oft die entscheidende Möglichkeit dar, einer höheren Schicht anzugehören als ihre Eltern (Esser 2006; King 2009).

Essentiell für die Positionierung am Arbeitsmarkt und innerhalb der sozialen Schichtung erscheint zudem der Erwerb der jeweiligen Sprache des Einwanderungslandes. Sprachdefizite erschweren die Nutzung des ansonsten vorhandenen Humankapitals (z.B. Fachausbildung) (Esser 2006; Riesenfelder et al. 2011). Ein Problem der Nutzbarmachung des eigenen Humankapitals stellt für die erste Generation des Weitereren die fehlende Anerkennung von im Herkunftsland erworbenen Bildungsabschlüssen dar (Nohl 2010; Riesenfelder et al. 2011). Die Anerkennung von Bildungstiteln aus EU-Mitgliedstaaten ist in der Regel einfacher als jene von Zertifikaten aus Drittstaaten. Doch selbst wenn Bildungsabschlüsse Migranten weniger gut vor Armut schützen als Personen ohne Migrationshintergrund (z. B. King 2009), so sind dennoch gerade mit geringer Bildung ausgestattete Migranten von größeren Armuts- und Abstiegsrisiken betroffen (Verwiebe 2010). Dies zeigt sich für Wien, wo es durch die Integration gut ausgebildeter neuer Migranten am Arbeitsmarkt zu einer Verdrängung traditioneller Einwanderungsgruppen kommt, deren Qualifikationen in der Regel geringer sind.

$\mathrm{Zu}$ den arbeitsmarktrelevanten Faktoren zählen schlussendlich auch Erwerbsintensität, berufliche Position und Branchenzugehörigkeit. Art und Ausmaß der Erwerbstätigkeit ist entscheidend dafür, ob ein Abrutschen in Armut - und somit in untere gesellschaftliche Schichten - verhindert werden kann (McKernan und Ratcliffe 2005). Viele Migranten finden sich jedoch in schlecht entlohnten Dienstleistungstätigkeiten wieder oder arbeiten in Branchen (Hotellerie, Bau), in denen die Reallohnentwicklung stagniert (Fritsch et al. 2018). ${ }^{6}$ Der Wiener Arbeitsmarkt hat sich in den letzten Jahrzehnten zudem durch den Zuwachs an Teilzeitbeschäftigungen und Formen atypischer Beschäftigungen stark verändert. Zusätzlich sind viele Migranten seit 2011 wachsender Konkurrenz durch grenzüberschreitende Pendler ausgesetzt, die in der Regel besser ausgebildet und aufgrund der zumeist niedrigeren Lebenserhaltungskosten in ihren Herkunftsländern (z. B. Ungarn, Slowakei) auch bereit sind, niedrigere Löhne zu akzeptieren (Verwiebe et al. 2017; Wiesböck et al. 2016). Sie verdrängten in Wien lebende, geringer qualifizierte Migranten vom Wiener Arbeitsmarkt und sorgten für einen Anstieg der Arbeitslosigkeit (Schiman 2018).

Zusammenfassend formulieren wir vor dem Hintergrund der hier dargestellten Veränderungsprozesse und Wirkmechanismen drei Hypothesen, die das Schrumpfen der migrantischen Mittelschicht Wiens betreffen. Hypothese $H 1$ hat die Veränderung der Migrationsströme zum Gegenstand: Wir nehmen an, dass der Rückgang der Mittelschicht vor allem auf den Abstieg von Migrantengruppen aus der Türkei und dem ehemaligen Jugoslawien ( $H$ la) sowie Personen aus Drittstaaten $(H I b)$

\footnotetext{
${ }^{6}$ Gerade in Österreich bestehen ausgeprägte Unterschiede zwischen Berufsgruppen und Branchen (Hermann und Flecker 2009). Die Niedriglohnbeschäftigung ist vor allem in der Landwirtschaft, in der Gastronomie, in personenbezogenen Diensten und im Handel stark verbreitet (Fritsch et al. 2014). So liegt zum Beispiel der durchschnittliche Bruttostundenlohn in der Chemie- oder Metallindustrie bei 19 bis $20 €$, in der Gastronomie oder im Handel hingegen nur bei 9 bis $12 €$ (EU-SILC Daten aus dem Jahr 2016, eigene Berechnungen).
} 
zurückführen ist, nicht aber auf neuere Zuwanderergruppen aus den EU-Mitgliedstaaten $\left(\begin{array}{ll}H & 1\end{array}\right)$. Letztere sollten aufgrund ihrer hohen Qualifizierung und des leichteren Arbeitsmarktzugangs weniger stark von den Folgen der Wirtschaftskrise und den Verdrängungseffekten am Wiener Arbeitsmarkt betroffen sein. Die Hypothesen 2 und 3 beziehen sich auf arbeitsmarktrelevante Faktoren: Wir gehen zum einen davon aus, dass Unterschiede nach Herkunftsgruppen zum größten Teil auf diese Faktoren zurückzuführen sind ( $\left.\begin{array}{ll}H & 2\end{array}\right) .{ }^{7}$ In anderen Worten: Nicht die Herkunftsregion an sich bewirkt die Zugehörigkeit zu unteren Gesellschaftsschichten, sondern vielmehr Faktoren wie das Fehlen einer passenden Ausbildung, mangelnde Sprachkenntnisse, höhere Erwerbslosigkeit oder die Tätigkeiten in bestimmten Branchen. Zum anderen nehmen wir in diesem Zusammenhang an, dass zwei Entwicklungen am städtischen Arbeitsmarkt entscheidend beeinflusst haben, wie sich die Schichtung verändert hat (H 3). Die steigende Relevanz der Humankapitalausstattung dürfte nur hochqualifizierte Migranten begünstigt und vor dem sozialen Abstieg bewahrt haben (H 3a), während der Anstieg prekärer Arbeitsverhältnisse (u. a. der Teilzeitbeschäftigung) ein Hauptgrund für das Schrumpfen der migrantischen Mittelschicht darstellen könnte $(H 3 b)$.

\section{Empirische Datenbasis und Methodik}

\subsection{Daten und Variablen}

Für die folgenden Analysen werden zwei Datensätze herangezogen: Leben und Lebensqualität in Wien (LLQW 2003) und Sozialwissenschaftliche Grundlagenforschung für Wien (SOWI 2013). Die Erhebungen fanden zwischen Mai und Oktober 2003 bzw. Oktober 2012 und Juli 2013 statt. Die Stichprobenziehung folgte einem RDD-Verfahren (Random Digit Dialing) unter Einbezug von Handynummern. Es wurden 8000 bzw. 8100 CATI-Interviews in deutscher Sprache und jeweils 300 CATI-Interviews in der Muttersprache von Personen aus der Türkei und Ex-Jugoslawien geführt. Die Daten sind für die Wiener Bevölkerung ab 15 Jahren repräsentativ und die Fallzahlen von Personen mit Migrationshintergrund mit 2081 Personen 2003 und 2490 Personen 2013 für regionale Umfragen sehr hoch. Die Schätzung der Schichtzugehörigkeit von im Ausland geborenen Personen weicht nur geringfügig von den mit EU-SILC erzielten Ergebnissen ab. ${ }^{8}$ Im Gegensatz zu EU-SILC erlauben die verwendeten Daten zu Wien detailliertere Betrachtungen nach Merkmalen wie der Herkunftsregion, Staatsbürgerschaft, Einbürgerung oder Sprachkenntnissen.

\footnotetext{
7 Unter arbeitsmarktrelevanten Faktoren verstehen wir zusammenfassend sowohl individuelle Charakteristika, die am Arbeitsmarkt von Bedeutung sind (z. B. Bildung) als auch Charakteristika der Beschäftigung selbst (Voll/Teilzeit, Branchenzugehörigkeit etc.).

8 Diesen Studien zufolge wurden 2003 rund $48 \%$ unteren Schichten, $40 \%$ der Mittelschicht und $12 \%$ einer oberen Schicht zugeordnet (nach EU-SILC waren es 2004 etwa 45, 43 und 12\%). Im Jahr 2013 umfasst die Mittelschicht 32\% (in EU-SILC 30\%), die untere Mittelschicht und die Unterschicht 21 bzw. $38 \%$ (in EU-SILC 21 und $36 \%$ ).
} 
Zur Messung der Schichtzugehörigkeit greifen wir auf ein etabliertes Konzept zurück (Atkinson und Brandolini 2011; Burkhardt et al. 2013; Grabka und Frick 2008). Ausgangspunkt ist das jährlich verfügbare Haushaltsnettoeinkommen, das nach Anzahl der im Haushalt lebenden Erwachsenen und Kinder gewichtet wird. Auf Basis dieses äquivalisierten Haushaltseinkommens wird der österreichweite Median gebildet. Der Mittelschicht werden in der Folge Personen zugeordnet, deren Einkommen bei mehr als 80, aber unter $140 \%$ des Medianeinkommens liegt (für das Medianeinkommen der Referenzjahre siehe Till-Tentschert et al. (2004) und Statistik Austria (2014a)). Oberhalb der Mitte findet sich die obere Mittelschicht (140 bis 200\% des Medianeinkommens) und die Oberschicht (über 200\% des Medianeinkommens). Dazu kommen die untere Mittelschicht (60 bis $80 \%$ des Medianeinkommens) und die Unterschicht oder Schicht der Armutsgefährdeten (unter 60\% des Medianeinkommens).

Migrationsbezogene Faktoren. Die Herkunft der befragten Personen wurde anhand des Geburtslandes der Person selbst (bei der 1. Generation) oder ihrer Eltern (bei der 2. Generation) festgestellt und in fünf Kategorien erfasst: (1) Türkei, (2) Nachfolgestaaten des ehemaligen Jugoslawien (inkl. den EU-Staaten Slowenien und Kroatien), (3) EU-Mitgliedstaaten ab 1995, (4) sonstige EU-Mitgliedstaaten der Osterweiterung 2004 oder 2007, (5) Drittstaaten. Eine Person wird dann zur 1. Generation gezählt, wenn sie selbst im Ausland geboren wurde. Die 2. Generation umfasst Kinder von zugewanderten Personen, die aber selbst in Österreich zur Welt gekommen sind (zumindest ein Elternteil im Ausland geboren). Bei der Staatsbürgerschaft der Befragten werden drei Kategorien differenziert: (a) österreichisch seit Geburt, (b) durch Einbürgerung oder (c) Staatsbürgerschaft einer anderen Nation. Im Jahr 2013 wurden zudem die Sprachkenntnisse jener Personen, deren Muttersprache nicht Deutsch ist, anhand zweier Fragen erhoben: (a) Können Sie in Deutsch sehr gut, gut, mittelmäßig, eher schlecht oder gar nicht sprechen? (b) Können Sie in Deutsch sehr gut, gut, mittelmäßig, eher schlecht oder gar nicht schreiben? (jeweils $1=$ sehr gut, 5=gar nicht). Die Kombination aus beiden Antworten wurde herangezogen, um die Sprachkenntnisse wie folgt zu kategorisieren: (a) schriftlich und mündlich sehr gut, (b) schriftlich und mündlich gut (Durchschnitt von 1,5 bis 2,5), (c) mittelmäßig bis schlecht (Durchschnitt höher als 2,5).

Arbeitsmarktrelevante Faktoren. Der höchste Schulabschluss wird in vier Kategorien erfasst: (a) kein Abschluss oder Pflichtschulabschluss, (b) Lehrausbildung oder berufsbildende mittlere Schule, (c) höhere allgemein oder berufsbildende Schule oder Meisterschule, (d) Universitäts- oder Fachhochschulabschluss. Die Erwerbsintensität wird für selbständig tätige Personen sowie abhängig beschäftigte Arbeitnehmer in den Kategorien Vollzeit (mehr als $36 \mathrm{~h}$ pro Woche) und Teilzeit (bis zu 36 Wochenstunden) erfasst. Bei nicht erwerbstätigen Personen wird zwischen Arbeitslosen, Personen in Pension (Rente), in Ausbildung (Auszubildende, Schüler, Studierende) und Sonstigen (Personen in Karenz, Hausmänner und Hausfrauen etc.) differenziert. Beim Berufsstatus wird zwischen freiberuflich tätigen Personen, selbständig tätigen Personen, kleinen und mittleren Angestellten, hochqualifizierten Angestellten, Facharbeitern und Hilfsarbeitern unterschieden. Nach Branchen differenziert wird (allerdings nur in der Erhebung 2013) zwischen (1) Fischerei, Landund Forstwirtschaft, (2) Energie-, Wasser- und Baugewerbe, (3) Handel, (4) Gastge- 
werbe, (5) Information und Kommunikation, (6) Finanzdienstleistungen sowie wirtschaftsnahe Dienstleistungen, (7) Öffentliche Verwaltung, Unterricht etc., (8) Gesundheits- und Sozialwesen, (9) Sonstige Dienstleistungen, (10) Anderes (inkl. verarbeitendes Gewerbe). Nachdem für Österreich Niedriglohnbeschäftigung häufig im Handel festgestellt wurde, dient dieser als Referenz, die mit anderen Wirtschaftsbereichen verglichen wird. Von Interesse für die Betrachtung sind dabei vor allem das Gastgewerbe, in dem ähnliche Bedingungen vorherrschen sollten, sowie die Finanzdienstleistungen und andere wirtschaftsnahe Bereiche, die ganz andere Beschäftigungsbedingungen aufweisen.

Das vorhandene Sample spiegelt einige wesentliche Entwicklungen der Zuwanderung und des Arbeitsmarktes in Wien. So hat vor allem auch der Anteil an Migranten aus den alten und neuen EU-Mitgliedstaaten zugenommen (von 19 auf 30 bzw. von 22 auf 29\%), während der Anteil der Personen aus dem ehemaligen Jugoslawien und der Türkei zurückging (von 28 auf 18 bzw. 18 auf 13\%). Migranten und ihre Kinder sind im Jahr 2013 im Durchschnitt höher gebildet als noch 2003. Allein der Anteil der Personen mit Hochschulabschluss stieg von 16 auf 29\% an. Auch andere Veränderungen auf dem Arbeitsmarkt zeigen sich im Sample, etwa ein Rückgang bei den Anteilen von Vollzeitbeschäftigten und Arbeitern, sowie ein Zuwachs sowohl bei niedrigqualifizierten als auch bei hochqualifizierten Angestellten (für Details s. Online-Anhang, Abschn. A2).

\subsection{Analysestrategie und Methoden}

Um Hypothese $H 1 \mathrm{zu}$ prüfen, werden in deskriptiven Analysen in einem ersten Schritt zwischen 2003 und 2013 stattgefundene Veränderungen im Schichtgefüge unter Personen mit Migrationshintergrund nach Herkunftsregionen analysiert. Zur Erklärung des Schrumpfens der Mittelschicht (Hypothese $H 1$ bis $H 3$ ) werden in der Folge logistische Regressionsmodelle geschätzt. In den Tabellen werden aus den Modellen resultierende Average Marginal Effects (AME) präsentiert, da diese am ehesten über verschiedene Modelle hinweg vergleichbar sind (siehe Best und Wolf 2012). Ein AME gibt den durchschnittlichen Effekt einer Variablen auf die Wahrscheinlichkeit einer bestimmten Schicht anzugehören wieder.

Um die Rolle der Herkunft der Migranten für die Veränderung der Schichtung genauer zu beleuchten (Hypothese $H$ 2), wird der Unterschied zwischen der Schichtzugehörigkeit von Migranten in den Jahren 2003 und 2013 im zweiten Schritt in einem multinomialen Modell untersucht, in dem zwischen unteren Schichten, der Mittelschicht und oberen Schichten unterschieden wird. Dafür wird ein gepooltes Sample der beiden Datensätze herangezogen. In dieses Modell geht zunächst nur eine Dummy-Variable, die den Unterschied zwischen 2003 und 2013 festhält, und die Herkunft ein (Modell a1). Danach werden weitere migrationsspezifische Größen (Generation, Staatsbürgerschaft), arbeitsmarktrelevante und arbeitsmarktbezogene Charakteristika (Schulabschluss, Erwerbsintensität, Berufsstatus) sowie Kontrollvariablen (Haushaltsform, Geschlecht, Alter) in das Modell aufgenommen (Modell a2 bis a4). Die Unterschiede nach Herkunftsgruppen müssten kleiner werden oder gänzlich verschwinden, wenn Unterschiede in den zusätzlich einbezogenen Größen (Bildung etc.) für diese verantwortlich sind. 
Im nächsten Schritt wird der zuletzt angesprochene Test separat für die Jahre 2003 und 2013 wiederholt. Zur Anwendung kommen diesmal allerdings binomiale Modelle, die nur zwischen der Zugehörigkeit zu einer unteren Schicht (unterhalb der Mittelschicht) oder anderen Schichten (ab der Mittelschicht) unterscheiden, da diese in weiterer Folge zur Durchführung statistischer Testverfahren (zur Absicherung der Befunde) sowie detaillierterer Dekompositionsanalysen herangezogen werden können. Die binomialen logistischen Modelle werden wiederum schrittweise aufgebaut: Zunächst wird als einziger Erklärungsfaktor die Herkunft der Personen mit Migrationshintergrund berücksichtigt (Modell b1). Danach werden zusätzlich weitere migrationsspezifische Charakteristika sowie Arbeitsmarktfaktoren in das Modell aufgenommen (Modell b2). Mithilfe der KHB-Methode ${ }^{9}$ wird nun jedoch explizit getestet, ob sich die festgestellten Unterschiede zwischen den Personen aus unterschiedlichen Herkunftsregionen verringern, wenn diese weiteren Variablen in das Modell aufgenommen werden (Modell b2). Mit der von Allison (1999) und Hoetker (2007) dargelegten Methode wird zudem getestet, ob sich die Koeffizienten in den separat für zwei Erhebungen geschätzten Modellen voneinander unterscheiden (für Details siehe den Online-Appendix, Abschn. A3). ${ }^{10}$ Darüber hinaus wird in einem separaten Modell auch die Rolle der Branchenzugehörigkeit und der Sprachkenntnisse (nur im Jahr 2013 abgefragt) für die Schichtzugehörigkeit beleuchtet.

Im letzten Schritt wird mittels Dekompositionsanalysen konkreter geprüft, welche Bedeutung die veränderte Zusammensetzung der Migrantenpopulation für die Entwicklung der migrantischen Mittelschicht hat. Präsentiert werden zunächst detaillierte Ergebnisse für die binomialen Modelle (b1 und b2), die anhand der Methode von Fairlie (2006) und Jann (2006) gewonnen wurden. Diese erlauben die Diskussion einzelner Faktoren (z. B. auch einzelner Herkunfts- oder Bildungskategorien). Mit der von Sinning et al. (2008) dargelegten Dekompositionsmethode wird danach geprüft, ob die wesentlichen Resultate der binomialen Modelle auch in multinomialen Modellen, die zwischen drei oder fünf Schichten unterscheiden, und bei anderer Modellspezifikation Bestand haben. Die beiden zuletzt dargelegten Analyseschritte (Test auf Unterschiede in den Koeffizienten und Dekomposition) erlauben schlussendlich Rückschlüsse auf die Bedeutung der in Hypothese $H 3$ vorgebrachten Veränderungen am Arbeitsmarkt.

\footnotetext{
${ }_{9}$ Bei der von Karlson et al. (2012) vorgeschlagenen Methode handelt es sich um eine Anwendung der Grundidee des bekannten Sobel-Tests zur Prüfung von Mediationen auf logistische Regressionsmodelle. Eine spezifische Methode ist notwendig, da Koeffizienten aus verschiedenen logistischen Modellen aufgrund möglicher Unterschiede in der Varianz der latenten abhängigen Variablen nicht direkt miteinander verglichen werden können.

${ }^{10}$ Um möglichen Problemen aufgrund von unbeobachteter Variation zu entgehen, wird dem Vorschlag Hoetkers (2007) gefolgt und anstatt einzelner Koeffizienten jeweils das Verhältnis zweier Koeffizienten getestet (mehr Information dazu im Online-Appendix, Abschn. A3).
} 
Sämtliche Analysen im Text beziehen sich auf Personen mit Migrationshintergrund. Analysen, in denen Unterschieden zwischen Einheimischen und Personen mit Migrationshintergrund nachgegangen wird, finden sich im Online-Appendix (Abschn. A3). ${ }^{11}$

\section{Ergebnisse: Was erklärt das Schrumpfen der migrantischen Mittelschicht?}

\subsection{Deskriptive Analysen zur Entwicklung der migrantischen Mittelschicht}

Tabelle 1 bildet die Entwicklung des migrantischen Schichtgefüges in Wien nach Herkunftsregion ab. Es zeigt sich zunächst erwartungsgemäß und in Einklang mit der Literatur (vgl. Stadt Wien 2014; Statistik Austria 2015), dass der Anteil der Mittelschicht bei Personen mit türkischem Migrationshintergrund am geringsten und bei Migranten aus EU-Mitgliedstaaten am höchsten ist. Während weniger als ein Fünftel der türkischen Migranten zur Mittelschicht zählt, gehören fast 50\% der EU-internen Migranten zur gesellschaftlichen Mitte. Die größten Veränderungen zwischen 2003 und 2013 zeigen sich für Personen aus den Nachfolgestaaten Jugoslawiens und aus Drittstaaten. Bei ihnen ging der Anteil der Mittelschicht jeweils um mehr als zehn Prozentpunkte zurück. Parallel dazu nahm der Anteil der Unterschicht in diesen Gruppen deutlich zu (für ähnliche Befunde zu Deutschland siehe Burkhardt et al. 2013; Tucci und Wagner 2005; Verwiebe 2010). Zwar kam es auch bei den EU-15 und EU-12 Migranten zu einer erkennbaren Abwärtsmobilität, doch fällt diese im Vergleich zu anderen Gruppen moderater aus.

Die verwendeten Daten zeigen auch für andere Indikatoren Polarisierungstendenzen auf und unterstreichen somit die bislang diskutierten Befunde. Tatsächlich kennzeichnet die Migranten mit türkischen Wurzeln im Vergleich zu den anderen Gruppen die höchsten Anteile an Personen, die lediglich über einen Pflichtschulabschluss verfügen (2003: 53\%, 2013: 34\%). Die Arbeitslosigkeit (15 bzw. 13\%) ist die höchste unter allen Migrantengruppen. Zudem ist bei den Türken auch der Anteil an Hilfsarbeitern, gemeinsam mit Personen aus dem ehemaligen Jugoslawien (2003 noch rund $30 \%$ ), deutlich größer. Diese beiden Gruppen sind außerdem überproportional häufig im Handel tätig, während Personen aus den EU-Mitgliedsstaaten häufiger in hochqualifizierten Angestelltenpositionen (z. B. rund $25 \%$ bei EU-15 im Jahr 2013) und im Wirtschafts- und Finanzdienstleistungssektor arbeiten. Etwas schwieriger sind Personen aus Drittstaaten einzuordnen. Sie sind zwar Großteils gut ausgebildet, aber auch seltener als andere Arbeitsmarktgruppen in Vollzeitjobs beschäftigt. Heraus sticht, dass bei ihnen der Anteil an Personen in kleinen und mittleren Angestelltenpositionen im Gegensatz zu den anderen Gruppen zwischen 2003

\footnotetext{
11 Kurz zusammengefasst sprechen diese Modelle und Tests dafür, dass (a) der Unterschied zwischen Personen mit und ohne Migrationshintergrund zu einem gewissen Teil, aber keineswegs vollständig auf Unterschiede bei Bildung, Branchenzugehörigkeit etc. zurückzuführen ist, und dass (b) die Effekte der einzelnen betrachteten Faktoren (Bildung, Berufsstatus etc.) auf die Schichtzugehörigkeit für Personen mit und ohne Migrationshintergrund vergleichbar sind.
} 
Tab. 1 Zugehörigkeit zu Schichten nach Herkunftsregion (Angaben in \%)

\begin{tabular}{llllllll}
\hline & & $\begin{array}{l}\text { Unter- } \\
\text { schicht }\end{array}$ & $\begin{array}{l}\text { Untere } \\
\text { Mittel- } \\
\text { schicht }\end{array}$ & $\begin{array}{l}\text { Mittel- } \\
\text { schicht }\end{array}$ & $\begin{array}{l}\text { Obere } \\
\text { Mittel- } \\
\text { schicht }\end{array}$ & $\begin{array}{l}\text { Ober- } \\
\text { schicht }\end{array}$ & $N$ \\
\hline Türkei & 2003 & 56 & 23 & 18 & 2 & 1 & 288 \\
& 2013 & 58 & 22 & 17 & 2 & $<0$ & 248 \\
Ex- & 2003 & 28 & 22 & 41 & 7 & 1 & 452 \\
Jugoslawien & 2013 & 37 & 26 & 29 & 6 & 1 & 341 \\
EU-15 & 2003 & 17 & 10 & 49 & 15 & 8 & 316 \\
& 2013 & 21 & 21 & 44 & 10 & 4 & 569 \\
EU-Andere & 2003 & 21 & 13 & 49 & 13 & 4 & 359 \\
& 2013 & 24 & 21 & 44 & 9 & 2 & 562 \\
Drittstaaten & 2003 & 28 & 12 & 44 & 13 & 3 & 229 \\
& 2013 & 40 & 20 & 32 & 6 & 2 & 198 \\
\hline
\end{tabular}

Quelle: LLQW 2003, SOWI 2013 (eigene Berechnungen; gewichtet).

und 2013 rückläufig war. Zudem befinden sich viele Personen dieser Gruppe in einer Ausbildung (für Details siehe Online-Appendix, Abschn. A2). Die nun folgenden multivariaten Analysen sollen u. a. dazu beitragen zu klären, inwieweit die Unterschiede bei der Schichtung zwischen den Migrantengruppen auf diese Unterschiede zurückzuführen sind.

\subsection{Multivariate Analysen der Schichtzugehörigkeit}

Die Tab. 2, 3 und 4 geben die Befunde mehrerer logistischer Regressionen wieder. Hier bestätigt sich zunächst anhand des gepoolten Samples (Tab. 2), dass die Wahrscheinlichkeit, der Mittelschicht anzugehören, im Vergleich zur Referenzgruppe der türkischen Migranten bei allen anderen Gruppen signifikant höher ist (vor allem bei Migranten aus EU-Mitgliedstaaten). Dasselbe gilt ebenso für die Zugehörigkeit zu einer Schicht oberhalb der Mittelschicht. Umgekehrt ist die Wahrscheinlichkeit, einer Schicht unterhalb der Mittelschicht anzugehören, bei Türken am höchsten. Letzteres bestätigen auch die separat für 2003 und 2013 durchgeführten binomialen Analysen (Tab. 3).

Ein Vergleich der Modelle a1 bis a4 weist zudem darauf hin, dass die Unterschiede nach Herkunftsregion kleiner werden, wenn andere migrationsspezifische und insbesondere Arbeitsmarktfaktoren in die Analyse einbezogen werden (Tab. 2). Die entsprechenden Koeffizienten sind auch in Modell b2 geringer als in Modell b1 (Tab. 3). Sämtliche KHB-Tests auf Unterschiede zwischen den Koeffizienten in den Modellen b1 und b2 zeigen ein signifikantes Ergebnis. Zumindest teilweise können die Unterschiede zwischen den Herkunftsgruppen also auf die zusätzlich einbezogenen Faktoren zurückgeführt werden. Damit bestätigt sich tendenziell unsere Hypothese $H 2$.

Im Detail demonstriert Modell b2 weiter die Relevanz von Arbeitsmarktfaktoren für die Schichtzugehörigkeit. Höhere Bildung, eine bessere berufliche Positionierung und größere Erwerbsintensität verringern die Chance, unteren Schichten anzugehören. Ergänzende Berechnungen (Tab. 4) verdeutlichen außerdem, dass sich die Bran- 
Tab. 2 Unterschiede der Schichtzugehörigkeit bei Personen mit Migrationshintergrund (Average Marginal Effects, AME)

\begin{tabular}{|c|c|c|c|c|c|c|c|c|}
\hline \multirow{2}{*}{$\begin{array}{l}\text { Zugehörigkeit zu } \\
\text { Herkunftsregion Ex-Jugoslawt } \\
\text { im Vergleich zur Türkei }\end{array}$} & \multicolumn{2}{|c|}{$\begin{array}{l}\text { Modell a1 } \\
\text { AME }\end{array}$} & \multicolumn{2}{|c|}{$\begin{array}{l}\text { Modell a2 } \\
\text { AME }\end{array}$} & \multicolumn{2}{|c|}{$\begin{array}{l}\text { Modell a3 } \\
\text { AME }\end{array}$} & \multicolumn{2}{|c|}{$\begin{array}{l}\text { Modell a4 } \\
\text { AME }\end{array}$} \\
\hline & & & & & & & & \\
\hline Unterhalb der Mitte & $-0,28$ & $* * *$ & $-0,25$ & $* * *$ & $-0,20$ & $* * *$ & $-0,18$ & $* * *$ \\
\hline Mitte der Gesellschaft & 0,19 & $* * *$ & 0,20 & $* * *$ & 0,15 & $* * *$ & 0,14 & $* * *$ \\
\hline Oberhalb der Mitte & 0,09 & $* * *$ & 0,05 & $* * *$ & 0,05 & $* *$ & 0,04 & $*$ \\
\hline \multicolumn{9}{|l|}{$\begin{array}{l}\text { Herkunftsregion EU-15 } \\
\text { im Vergleich zur Türkei }\end{array}$} \\
\hline Unterhalb der Mitte & $-0,48$ & $* * *$ & $-0,45$ & $* * *$ & $-0,29$ & $* * *$ & $-0,24$ & $* * *$ \\
\hline Mitte der Gesellschaft & 0,28 & $* * *$ & 0,29 & $* * *$ & 0,19 & $* * *$ & 0,17 & $* * *$ \\
\hline Oberhalb der Mitte & 0,20 & $* * *$ & 0,16 & $* * *$ & 0,10 & $* * *$ & 0,08 & $* * *$ \\
\hline \multicolumn{9}{|l|}{$\begin{array}{l}\text { Herkunftsregion EU-Andere } \\
\text { im Vergleich zur Türkei }\end{array}$} \\
\hline Unterhalb der Mitte & $-0,43$ & $* * *$ & $-0,38$ & $* * *$ & $-0,26$ & $* * *$ & $-0,21$ & $* * *$ \\
\hline Mitte der Gesellschaft & 0,26 & $* * *$ & 0,27 & $* * *$ & 0,18 & $* * *$ & 0,15 & $* * *$ \\
\hline Oberhalb der Mitte & 0,16 & $* * *$ & 0,12 & $* * *$ & 0,08 & $* * *$ & 0,06 & $* * *$ \\
\hline \multicolumn{9}{|l|}{$\begin{array}{l}\text { Herkunftsregion Drittstaaten } \\
\text { im Vergleich zur Türkei }\end{array}$} \\
\hline Unterhalb der Mitte & $-0,32$ & $* * *$ & $-0,28$ & $* * *$ & $-0,18$ & $* * *$ & $-0,16$ & $* * *$ \\
\hline Mitte der Gesellschaft & 0,18 & $* * *$ & 0,18 & $* * *$ & 0,13 & $* * *$ & 0,11 & $* * *$ \\
\hline Oberhalb der Mitte & 0,14 & $* * *$ & 0,10 & $* * *$ & 0,06 & $* *$ & 0,05 & $*$ \\
\hline \multicolumn{9}{|l|}{ Kontrollvariablen: } \\
\hline $\begin{array}{l}\text { Staatsbürgerschaft, } \\
\text { 1./2. Generation }\end{array}$ & - & & Inkl & & Inkl & & Inkl & \\
\hline $\begin{array}{l}\text { Bildung, Erwerbstätigkeit, } \\
\text { Berufsstatus }\end{array}$ & - & & - & & Inkl & & Inkl & \\
\hline $\begin{array}{l}\text { Haushaltsform, Geschlecht, } \\
\text { Alter }\end{array}$ & - & & - & & - & & Inkl & \\
\hline \multicolumn{9}{|l|}{2013 im Vergleich zu 2003} \\
\hline Unterhalb der Mitte & 0,08 & $* * *$ & 0,08 & $* * *$ & 0,12 & $* * *$ & 0,11 & $* * *$ \\
\hline Mitte der Gesellschaft & $-0,04$ & $*$ & $-0,04$ & $* *$ & $-0,07$ & $* * *$ & $-0,06$ & $* * *$ \\
\hline Oberhalb der Mitte & $-0,04$ & * & $-0,04$ & $* * *$ & $-0,05$ & $* * *$ & $-0,05$ & $* * *$ \\
\hline Konstante & Inkl & & Inkl & & Inkl & & Inkl & \\
\hline Adj. Count $\mathrm{R}^{2}$ & 9 & & 9 & & 20 & & 25 & \\
\hline
\end{tabular}

Quelle: LLQW 2003, SOWI 2013 (eigene Berechnungen; ungewichtet); $N=3557$

(*) $p<0,10 ; * p<0,05 ; * * p<0,01 ; * * * p<0,001$

chenzugehörigkeit wie erwartet auswirkt: Die Tätigkeit in einem hochspezialisierten Bereich, wie den Finanzdienstleistungen und anderen wirtschaftsnahen Dienstleistungen, führt im Gegensatz zu einer Beschäftigung im Handel oder im Gastgewerbe zu deutlich besseren Chancen, nicht einer Gesellschaftsschicht unterhalb der Mitte zugeordnet zu werden. Diese Befunde befinden sich im Einklang mit der bestehenden Literatur (z.B. Abrassart 2013; Mohr 2007; Vandecasteele 2011) und unserer 
Tab. 3 Einflussfaktoren auf die Zugehörigkeit zu unteren Schichten bei Personen mit Migrationshintergrund in den Jahren 2003 und 2013 im Vergleich

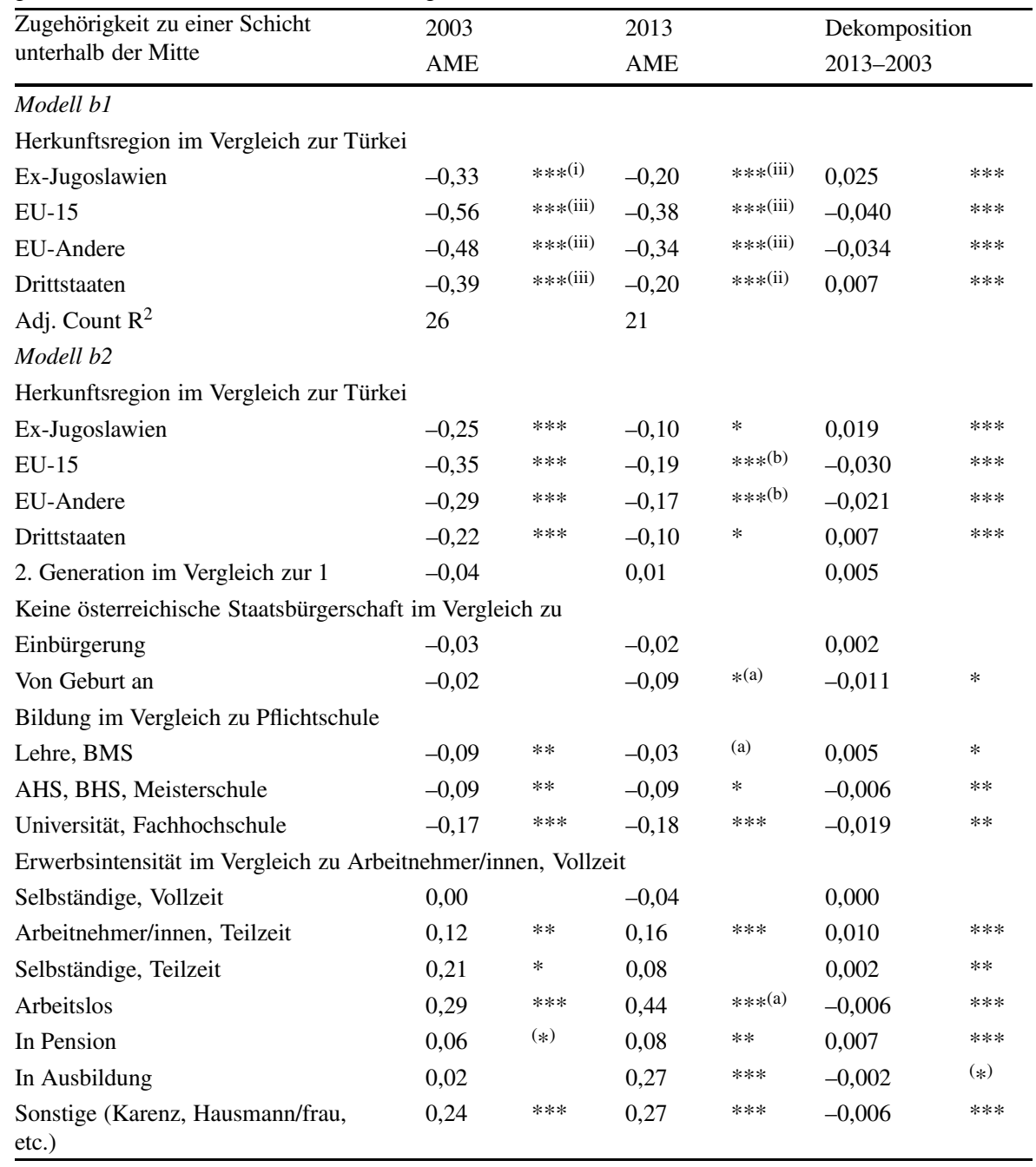

theoretischen Argumentation (Hypothese $H$ 3), dass die Arbeitsmarktentwicklung zu Veränderungen im Schichtgefüge geführt haben könnte.

Darüber hinaus deuten die Befunde in Tab. 3 darauf hin, dass die Integration in die Aufnahmegesellschaft in wirtschaftlichen Krisenzeiten an Bedeutung gewinnt. Menschen mit Migrationshintergrund, die von Geburt an im Besitz der österreichischen Staatsbürgerschaft sind, gehören 2013, aber noch nicht 2003 signifikant seltener unteren Schichten an (ähnliche Befunde finden sich bei Herzog-Punzenberger 2017, S. 235). Die Ergebnisse in Tab. 4 bestätigen zudem, dass die Sprachkenntnisse mitentscheidend dafür sein dürften, in höhere Schichten aufzusteigen. Je geringer sie sind, desto größer ist die Wahrscheinlichkeit, den unteren Schichten anzugehören. 
Tab. 3 (Fortsetzung)

\begin{tabular}{|c|c|c|c|c|c|c|}
\hline \multirow{2}{*}{$\begin{array}{l}\text { Zugehörigkeit zu einer Schicht } \\
\text { unterhalb der Mitte }\end{array}$} & \multicolumn{2}{|l|}{2003} & \multicolumn{2}{|l|}{2013} & \multicolumn{2}{|c|}{ Dekomposition } \\
\hline & \multicolumn{2}{|l|}{ AME } & \multicolumn{2}{|l|}{ AME } & \multicolumn{2}{|c|}{ 2013-2003 } \\
\hline \multicolumn{7}{|c|}{ Berufsstatus im Vergleich zu kleinen und mittleren Angestellten } \\
\hline Freiberuflich tätig & 0,01 & & 0,02 & & 0,000 & \\
\hline Selbständig tätig & 0,00 & & 0,03 & & 0,000 & \\
\hline Hochqualifizierte Angestellte & $-0,14$ & $* * *$ & $-0,13$ & $* * *$ & $-0,010$ & $* * *$ \\
\hline Facharbeiter/innen & 0,11 & $* *$ & 0,06 & & $-0,001$ & $*$ \\
\hline Hilfsarbeiter/innen & 0,20 & $* * *$ & 0,27 & $* * *$ & $-0,018$ & $* * *$ \\
\hline Sonstige (inkl. unbekannt) & 0,14 & $*$ & 0,00 & & $-0,001$ & \\
\hline Adj. Count $\mathrm{R}^{2}$ & 41 & & 36 & & & \\
\hline$N$ & 1644 & & 1913 & & 3557 & \\
\hline
\end{tabular}

Die ersten beiden Spalten zeigen Average Marginal Effects (AME) für Personen mit Migrationshintergrund für das Jahr 2003 und das Jahr 2013. In der letzten Spalte dargestellt wird das Ergebnis der Dekompositionsanalyse nach Fairlie (2006) und Jann (2006). Als Basismodell dient das gepoolte Sample. Durchgeführt wurde ein Bootstrap mit 1000 Replikationen. Zusätzlich wurde die Anordnung der Variablen randomisiert. ${ }^{(*)} p<0,10 ; * p<0,05 ; * * p<0,01 ; * * * p<0,001$

KHB-Tests auf Unterschiede zwischen Modell b1 und b2 (bei Modell b1 gekennzeichnet): (i) $p<0,05$; (ii) $p<0,01$; (iii) $p<0,001$

Ratio-Tests auf Unterschiede zwischen den Koeffizienten 2003 und 2013 (nur für Modell b2): (a) $p<0,01$; (b) $p<0,001$

Quelle: LLQW 2003, SOWI 2013 (eigene Berechnungen; ungewichtet)

Mit einem Blick (zurück) auf Tab. 2 zeigt sich jedoch auch, dass weder Veränderungen in den migrationsspezifischen noch in den arbeitsmarktrelevanten Charakteristika der migrantischen Population die beobachtete Dynamik zur Gänze erklären können. Der Unterschied zwischen den beiden Erhebungsjahren 2003 und 2013 bleibt auch unter Einbezug dieser Faktoren bestehen (Modell a1 bis a4). Dass der Unterschied in den Modellen a3 und a4 sogar etwas größer als in den Modellen a1 und a2 ist, könnte bedeuten, dass das Schrumpfen der Mittelschicht sogar stärker ausgefallen wäre, wenn die Migranten 2013 ein ähnliches Bildungsniveau wie 2003 aufweisen würden. Dekompositionsanalysen können dies weiter aufklären (siehe Abschn. 4.3).

\subsection{Dekompositionsanalysen zur Veränderung im Schichtgefüge}

Um die Frage, wie sich die Veränderung der Migrationsdynamik und damit der Zusammensetzung der migrantischen Bevölkerung in Wien auf die Schichtzugehörigkeit ausgewirkt hat, abschließend zu beantworten, werden Befunde aus Dekompositionsanalysen herangezogen. Tabelle 5 fasst die Hauptergebnisse verschiedenster Dekompositionsanalysen zusammen, die sich auf unterschiedliche Schichtungsvariablen (zweistufig wie in Tab. 3 bis zu fünfstufig wie in Tab. 1) und Modellspezifikationen beziehen. Sämtliche Befunde in der Tabelle verdeutlichen, dass die Veränderung in der Zusammensetzung der Migrantenpopulation (Komposition der Sample-Charakteristika) einen ungleichheitsreduzierenden Effekt hatte. Sie dämpfte den Anstieg der Armut und wirkte somit dem Schrumpfen der migrantischen 
Tab. 4 Die Rolle von Sprachkenntnissen und Branche für die Schichtzugehörigkeit bei Personen mit Migrationshintergrund 2013 (Average Marginal Effects, AME)

\begin{tabular}{lll}
\hline Zugehörigkeit zu einer Schicht unterhalb der Mitte & AME \\
\hline Migrationshintergrund $(M H):$ 1. Generation & Ref & 0,02 \\
2. Generation & Ref & $*$ \\
Sprachkenntnisse: Deutsch Muttersprache & 0,10 & $* *$ \\
Schriftlich und mündlich sehr gut & 0,13 & $* * *$ \\
Gut (Durchschnittsnote 1,5-2,5) & 0,19 & $*, 06$ \\
Mittelmäßig bis schlecht (Note 3-5) & Ref \\
Unbekannt & 0,10 & $*$ \\
Branche (Auswahl): Handel & 0,10 \\
Gastgewerbe & $-0,16$ \\
Land- u. Forstwirtschaft, Fischerei & 0,06 \\
Finanz-, wirtschaftsnahe Dienstleistung & 43 \\
Sonstige Dienstleistungen & 1861 \\
$N$ Adj. $R^{2}$ & & \\
\hline
\end{tabular}

Nicht dargesetllt werden die Konstante sowie die Kontrollvariablen Bildung, Erwerbsintensität, Berufsstatus, Haushaltsform, Geschlecht und Alter. Neben den abgebildeten Branchen wurde zudem auch auf andere Wirtschaftsbereiche kontrolliert. ${ }^{(*)} p<0,10 ; * p<0,05 ; * * p<0,01 ; * * * p<0,001$

Quelle: SOWI 2013 (eigene Berechnungen; ungewichtet)

Mittelschicht entgegen. Wir sprechen in diesem Zusammenhang von vorteilhaften Kompositionseffekten.

Woraus ergaben sich nun kompositionell vorteilhafte Veränderungen? Die detaillierten Befunde aus Tab. 3 (letzte Spalte) zeigen etwa, dass die Zunahme des Anteils von Migranten aus den EU-Staaten innerhalb der Bevölkerung einen ungleichheitsreduzierenden Effekt hatte. Da es 2013 anteilig deutlich mehr Migranten aus EU-Staaten als 2003 gibt, wirkt sich die Zusammensetzung der Bevölkerung mit Migrationshintergrund positiv (d.h. vorteilhaft) auf die Risiken aus, der unteren Mittelschicht oder der Unterschicht anzugehören: Unter sonst gleichen Bedingungen würden die Schichten unterhalb der Mittelschicht allein aufgrund der vorteilhafteren Verteilung nach Herkunftsregionen schrumpfen. Daraus, dass innerhalb der migrantischen Bevölkerung Wiens der Anteil von Personen stark zunimmt, die seit Geburt die österreichische Staatsbürgerschaft besitzen, und dass der Anteil der gut qualifizierten und hoch gebildeten Migranten ansteigt, resultieren ebenfalls kompositionell vorteilhafte Entwicklungen. Auch die Zunahme der Beschäftigung von hochqualifizierten Angestellten und der Rückgang von Hilfsarbeitertätigkeiten zwischen 2003 und 2013 haben schließlich das Anwachsen der unteren Mittelschicht und der Unterschicht eher gedämpft. Diese vorteilhaften Kompositionseffekte haben kompositionell negative Veränderungen übertroffen. Mit Blick auf die Erwerbsintensität deuten die Befunde nämlich auch darauf hin, dass z. B. die Zunahme der Teilzeitbeschäftigung innerhalb des Untersuchungszeitraums für eine Erhöhung der Armutsgefährdung gesorgt hat. 
Tab. 5 Veränderung der Schichtzugehörigkeit bei Personen mit Migrationshintergrund

\begin{tabular}{|c|c|c|c|c|c|c|c|c|}
\hline \multirow{3}{*}{$\begin{array}{l}\text { Dekompositions- } \\
\text { analysen } \\
(\text { Auswahl }) \\
\text { Modell } 2 \text { aus Tab }\end{array}$} & \multicolumn{2}{|c|}{ Veränderung } & \multicolumn{2}{|c|}{$\begin{array}{l}\text { Sample-Cha- } \\
\text { rakteristika }\end{array}$} & \multicolumn{2}{|c|}{ Koeffizienten } & \multirow[t]{2}{*}{$\begin{array}{l}\text { Regressions- } \\
\text { modell }\end{array}$} & \multirow[t]{2}{*}{$\begin{array}{l}\text { Abhängige } \\
\text { Variable }\end{array}$} \\
\hline & 3 (Basisn & odell & ooltes Sam & $(e)^{\mathrm{a}}$ & & & & \\
\hline & 0,0311 & & $-0,0736$ & & 0,1047 & & Logistisch & Dichotom \\
\hline \multicolumn{9}{|c|}{ Alternative Dekompositionsanalysen (ausgewählte Varianten) ${ }^{\mathrm{b}}$} \\
\hline $\begin{array}{l}\text { (1) Basis- } \\
\text { modell } 0\end{array}$ & 0,0311 & (*) & $-0,0899$ & $* * *$ & 0,1211 & $* * *$ & Logistisch & Dichotom \\
\hline $\begin{array}{l}\text { (2) Basis- } \\
\text { modell } 0\end{array}$ & 0,0311 & * & $-0,0899$ & $* * *$ & 0,1211 & $* * *$ & Logistisch & Dichotom \\
\hline $\begin{array}{l}\text { (3) Basis- } \\
\text { modell } 1\end{array}$ & 0,0311 & (*) & $-0,0898$ & $* * *$ & 0,1210 & $* * *$ & Logistisch & Dichotom \\
\hline $\begin{array}{l}\text { (4) Basis- } \\
\text { modell } 1\end{array}$ & 0,0311 & * & $-0,0898$ & $* * *$ & 0,1210 & $* * *$ & Logistisch & Dichotom \\
\hline \multicolumn{9}{|c|}{ Varianten bei differenzierterer Berücksichtigung der Schichtung ${ }^{\mathrm{c}}$} \\
\hline $\begin{array}{l}\text { (5) Basis- } \\
\text { modell } 0\end{array}$ & 0,1228 & $*$ & $-0,2446$ & $* * *$ & 0,3675 & $* *$ & Ordinal & Dreistufig \\
\hline $\begin{array}{l}\text { (6) Basis- } \\
\text { modell } 1\end{array}$ & 0,1228 & $*$ & $-0,1733$ & $* * *$ & 0,2962 & $* * *$ & Ordinal & Dreistufig \\
\hline $\begin{array}{l}\text { (7) Basis- } \\
\text { modell } 0\end{array}$ & 0,0969 & (*) & $-0,3732$ & $* *$ & 0,4701 & $* *$ & Ordinal & Fünfstufig \\
\hline $\begin{array}{l}(8) \text { Basis- } \\
\text { modell } 1\end{array}$ & 0,0969 & $(*)$ & $-0,2632$ & $* * *$ & 0,3600 & $* * *$ & Ordinal & Fünfstufig \\
\hline
\end{tabular}

Quelle: LLQW 2003, SOWI 2013 (eigene Berechnungen; ungewichtet); $N=3557$

(*) $p<0,10 ; * p<0,05 ; * * p<0,01 ; * * * p<0,001$

a Abweichend von den anderen Dekompositionsanalysen wurde hier die Methode nach Fairlie (2006) und Jann (2006) angewandt (für Details siehe Tab. 3)

${ }^{b}$ Dargestellt werden verschiedene Varianten der Dekompositionsanalyse nach Sinning et al. (2008) bei jeweils 100 Replikationen. Die Dekompositionsanalysen beziehen dieselben unabhängigen Variablen ein wie das in Tab. 3 dargestellte Modell 2

${ }^{\mathrm{c}}$ Die logistischen Regressionsmodelle beziehen sich auf eine dichotome Variable, die die Zugehörigkeit zu einer Schicht unterhalb der Mitte abbildet. Ordinal logistische Regressionsmodelle differenzieren bei der Schichtzugehörigkeit genauer (dreistufig: Zugehörigkeit zu einer oberen Schicht, zur Mittelschicht oder einer unteren Schicht; fünfstufig: Oberschicht, obere Mittelschicht, Mittelschicht, untere Mittelschicht, Unterschicht)

Allerdings ergaben sich zusätzlich zu negativen Kompositionseffekten Veränderungen in den Effekten (den Koeffizienten) der arbeitsmarktrelevanten Faktoren, die zu einem Schrumpfen der Mittelschicht und einem Anwachsen der unteren Schichten geführt haben. ${ }^{12}$ Die veränderte Wirkung einzelner Faktoren deutet auf grundlegende Änderungen am Wiener Arbeitsmarkt hin (siehe Modell b2 in Tab. 3). So schützt z.B. ein Lehrabschluss 2013 nicht mehr gleichermaßen vor Zugehörigkeit zu den unteren Schichten wie noch 2003 (vgl. Mohr 2007; Vandecasteele 2011). Im

\footnotetext{
12 Konkret zeigt sich in Tab. 5 etwa für Modell b2 aus Tab. 3, dass die unteren sozialen Schichten innerhalb des Untersuchungszeitraums um gut 3 Prozentpunkte gewachsen sind. Der Anteil der migrantischen Bevölkerung in den unteren sozialen Schichten hätte durch die kompositionell vorteilhaften Veränderungen in der Zusammensetzung der Migrationsbevölkerung (z. B. durch die Zunahme von EU-Bürgern) allerdings um mehr als 7 Prozentpunkte sinken müssen. Dem steht jedoch die Veränderung bei den Koeffizienten entgegen, die stark ungleichheitserhöhende Effekte mit sich brachte (+10 Prozentpunkte).
} 
Jahr 2013 haben auch Arbeitslose und Auszubildende gegenüber vollzeitbeschäftigten Arbeitnehmern ein erhöhtes Risiko der unteren Mittelschicht oder der Schicht der Armutsgefährdeten anzugehören. Menschen mit Migrationshintergrund, die als Hilfsarbeiter tätig sind, haben nicht nur besonders hohe, sondern auch im Zeitverlauf zunehmende Risiken, den unteren sozialen Schichten anzugehören (ähnliche Befunde für Deutschland zeigte Geißler 2014, S. 292f.). In der Summe sprechen diese Befunde daher für Hypothese $H$ 3: Es sind Entwicklungen am Arbeitsmarkt, die entscheidend zum Schrumpfen der migrantischen Mittelschicht beigetragen haben.

Zusammenfassend kann festgehalten werden, dass unsere Analysen fünf Schlussfolgerungen nahelegen: (1) Alle Migrantengruppen sind in ökonomisch schwierigeren Zeiten von sozialem Abstieg betroffen. (2) Vom Schrumpfen der migrantischen Mittelschicht in Wien sind aber vor allem Personen aus dem ehemaligen Jugoslawien und aus Drittstaaten betroffen. (3) Dieses Schrumpfen wäre ohne die hochqualifizierte Zuwanderung aus alten und neuen EU-Mitgliedstaaten noch deutlicher ausgefallen. (4) Unterschiede zwischen den Migrantengruppen sind zu einem guten Teil auf deren Charakteristika (Bildung, Beschäftigungsausmaß, Berufsposition) zurückzuführen. (5) In erster Linie dürften Veränderungen am Arbeitsmarkt zu den negativen Veränderungen im Schichtgefüge geführt haben - wie etwa die Zunahme an Teilzeitbeschäftigung oder der abnehmende Schutz vor dem sozialen Abstieg durch eine mittlere berufliche Ausbildung indizieren.

\section{Diskussion}

Städte sind zumeist Ausgangs- und Brennpunkte sozialen Wandels und auch Veränderungen in der Arbeitswelt und Migrationsbewegungen konzentrieren sich häufig auf Städte. Der städtische Arbeitsmarkt ist mittlerweile nahezu vollständig ein Dienstleistungsarbeitsmarkt, mit dem eine starke Polarisierung der Berufs- und Einkommensstruktur einhergeht (Burgers und Musterd 2002; Goebel et al. 2012; Sassen 2016). Migration trägt zu dieser Polarisierung entscheidend bei (McDowell et al. 2009; Tai 2006). Dennoch mangelt es bisher an Studien, die der Bedeutung der Migration für Veränderungen im städtischen Schichtgefüge systematisch nachgehen (Bailey et al. 2017; Sanderson et al. 2015). Der vorliegende Beitrag versuchte zum Schließen dieser Forschungslücke beizutragen, indem er das Schrumpfen der migrantischen Mittelschicht exemplarisch für den Fall der Stadt Wien untersucht hat.

Unsere Analysen verdeutlichen erstens, dass das Schrumpfen der migrantischen Mittelschicht in den 2000er Jahren alle Zuwanderergruppen erfasste, die nicht ohnehin bereits zum größten Teil unteren Schichten angehörten. Migranten waren und sind am Arbeitsmarkt in der Regel benachteiligt: Sie arbeiten häufiger in Branchen mit geringen Löhnen, werden öfter in prekäre Arbeitsverhältnisse gedrängt und sind größeren Unsicherheiten ausgesetzt, da sie über geringere Sozialleistungsansprüche verfügen (Kalter und Granato 2018; Leschke und Galgögczi 2015). Es verwundert daher nicht, dass sie in Zeiten wirtschaftlicher Umwälzungen vermehrt in untere Schichten abrutschen, denn Migranten waren von Deindustrialisierung, Prekarisie- 
rung und nicht zuletzt den Konsequenzen der Finanzkrise in Europa mit am stärksten negativ betroffen (Europäische Kommission 2016).

Die Analysen bestätigten im Einklang mit den Annahmen der Stadtforschung (Friedmann 1986; McDowell et al. 2009; Sassen 1991; Tai 2006) und unserer ersten Hypothese zweitens, dass verschiedene Herkunftsgruppen unterschiedliche Positionen im Schichtgefüge der Stadt einnehmen. Nicht jede Gruppe war gleichermaßen vom sozialen Abstieg betroffen. In erster Linie gehören Personen aus der Türkei, den Nachfolgestaaten des ehemaligen Jugoslawiens und aus Drittstaaten prekären und armutsgefährdeten Schichten an. Tatsächlich sind Migranten in Wien allerdings heute zu größeren Anteilen EU-Bürger und deutlich höher gebildet als noch vor zwei Jahrzehnten (Stadt Wien 2016b; Verwiebe et al. 2015). Unsere multivariaten Berechnungen weisen darauf hin, dass dies für die Entwicklung der sozialen Schichtung äußerst relevant ist! Die migrantische Mittelschicht wäre noch viel stärker geschrumpft, wenn es diese Europäisierung der Zuwanderung nicht gegeben hätte.

Es zeigt sich zudem (vgl. Hypothese $H$ 2), dass ein guter Teil der Unterschiede zwischen den Migrantengruppen mit Faktoren wie der Bildung und der Arbeitsmarktintegration (Ausmaß der Erwerbstätigkeit etc.) zusammenhängt. Ein Teil der Unterschiede dürfte jedoch auch auf die unterschiedliche Nähe zur Kultur der Aufnahmegesellschaft und unterschiedlicher Betroffenheit von Diskriminierung zurückzuführen sein (Verwiebe et al. 2016). Diese Faktoren konnten wir in unserer Studie nicht abbilden. Wir konnten jedoch zusätzlich anhand der Daten der zweiten Erhebung zeigen, dass Sprachkenntnisse als „Schlüsselkompetenz“ (Esser 2006; Riesenfelder et al. 2011) die Schichtzugehörigkeit von Personen mit Migrationshintergrund wesentlich beeinflussen.

Drittens implizieren einige Befunde unserer Analysen, dass Veränderungen am Arbeitsmarkt maßgeblich zum Abstieg der migrantischen Mittelschicht geführt haben. Dies hat unter anderem damit zu tun, dass immer mehr Migranten in Teilzeitbeschäftigung arbeiten (müssen) und mittlere berufliche Ausbildungen zu weniger stabilen Erwerbsverläufen führen. Damit bestätigt sich auch unsere dritte Hypothese. Unsere Studie stimmt in dieser Hinsicht mit einer Vielzahl an Untersuchungen überein, die den Wandel von der Industrie- zur Dienstleistungsgesellschaft und im Besonderen die Zunahme prekärer Niedriglohnbeschäftigung als wesentliche Ursache der Zunahme sozialer Ungleichheit betrachten (Dallinger 2013; Lucifora et al. 2005).

Funding Open access funding provided by University of Vienna.

Open Access Dieser Artikel wird unter der Creative Commons Namensnennung 4.0 International Lizenz (http://creativecommons.org/licenses/by/4.0/deed.de) veröffentlicht, welche die Nutzung, Vervielfältigung, Bearbeitung, Verbreitung und Wiedergabe in jeglichem Medium und Format erlaubt, sofern Sie den/die ursprünglichen Autor(en) und die Quelle ordnungsgemäß nennen, einen Link zur Creative Commons Lizenz beifügen und angeben, ob Änderungen vorgenommen wurden.

\section{Literatur}

Abrassart, Aurélien. 2013. Cognitive skills matter: The employment disadvantage of low-educated workers in comparative perspective. European Sociological Review 29:707-719. 
Acemoglu, Daron. 2002. Technical change, inequality, and the labor market. Journal of Economic Literature 40:7-72.

Alderson, Arthur S., Jason Beckfield und François Nielsen. 2005. Exactly how has income inequality changed? Patterns of distributional change in core societies. International Journal of Comparative Sociology 46:405-423.

Allison, Paul D. 1999. Comparing logit and probit coefficients across groups. Sociological Methods \& Research 28:186-208.

AMS. 2016. Der Arbeitsmarkt für AusländerInnen. Vienna: AMS.

Aschauer, Alexandra, Ursula Ganal, Gerald Sirlinger, Peter Stanzl, Bettina Steffel und Sonja Österreicher. 2012. Wiener Sozialbericht 2012. Wien: Stadt Wien, Magistratsabteilung 24 - Gesundheits- und Sozialplanung.

Atkinson, Anthony B., und Andrea Brandolini. 2011. On the identification of the "middle class". Working Papers ECINEQ 217.

Autor, David H., Lawrence F. Katz und Melissa S. Kearney. 2006. The polarization of the U.S. labor market. NBER Working Paper No. 11986.

Bailey, Nick, Wouter P.C. van Gent und Sako Musterd. 2017. Remaking urban segregation: Processes of income sorting and neighbourhood change. Population, Space and Place 23:1-16.

Barone, Carlo, und Herman G. van de Werfhorst. 2011. Education, cognitive skills and earnings in comparative perspective. International Sociology 26:483-502.

Bauer, Werner T. 2008. Zuwanderung nach Österreich [Immigration to Austria]. Vienna: ÖGPP.

Beaverstock, Jonathan V. 2005. Transnational elites in the city: British highly-skilled inter-company transferees in New York city's financial district. Journal of Ethnic and Migration Studies 31:245-268.

Best, Henning, und Christof Wolf. 2012. Modellvergleich und Ergebnisinterpretation in Logit und ProbitRegressionen. Kölner Zeitschrift für Soziologie und Sozialpsychologie 64:377-395.

Böhnke, Petra. 2010. Hoher Flug, tiefer Fall? Abstiege aus der gesellschaftlichen Mitte und ihre Folgen für das subjektive Wohlbefinden. In Dynamiken (in) der gesellschaftlichen Mitte, Hrsg. Berger, Peter A. und Nicole Burzan, 231-249. Wiesbaden: VS Verlag für Sozialwissenschaften.

Boterman, Willem R., Dorien Manting und Sako Musterd. 2018. Understanding the social geographies of urban regions through the socio-economic and cultural dimension of class. Population, Space and Place 24:e2107.

Buera, Francisco J., und Joseph P. Kaboski. 2012. The rise of the service economy. American Economic Review 102:2540-2569.

Burgers, Jack, und Sako Musterd. 2002. Understanding urban inequality: a model based on existing theories and an empirical illustration. International Journal of Urban and Regional Research 26:403-413.

Burkhardt, Christoph, Markus Grabka, Olaf Groh-Samberg, Yvonne Lott und Steffen Mau. 2013. Mittelschicht unter Druck? Gütersloh: Bertelsmann Stiftung.

Butler, Tim. 2003. Gentrification and the middle classes. Aldershot: Ashgate.

Butler, Tim, Chris Hamnett und Mark Ramsden. 2008. Inward and upward: Marking out social class change in London, 1981-2001. Urban Studies 45:67-88.

Cunningham, Niall, und Mike Savage. 2017. An intensifying and elite city. New geographies of social class and inequality in contemporary London. City 21:25-46.

Dallinger, Ursula. 2013. The endangered middle class? A comparative analysis of the role played by income redistribution. Journal of European Social Policy 23:83-101.

Davidson, Mark, und Elvin Wyly. 2012. Class-ifying London: Questioning social division and space claims in the post-industrial metropolis. City 16:395-421.

Dietze, Gabriele. 2009. Okzidentalismuskritik. Möglichkeiten und Grenzen einer Forschungsperspektivierung. In Kritik des Okzidentalismus. Transdisziplinäre Beiträge zu (Neo-) Orientalismus und Geschlecht, Hrsg. Dietze, Gabriele, Claudia Brunner und Edith Wenzel, 23-54. Bielefeld: Transcript.

Esping-Andersen, Gøsta. 2007. Sociological explanations of changing income distributions. American Behavioral Scientist 50:639-658.

Esser, Hartmut. 2006. Sprache und Integration: Die sozialen Bedingungen und Folgen des Spracherwerbs von Migranten. Frankfurt a.M.: Campus.

Europäische Kommission. 2016. Migrant integration statistics-employment. http://ec.europa.eu/eurostat/ statistics-explained/index.php/Migrant_integration_statistics_-_employment (Zugegriffen: 04.07. 2016).

Ewers, Michael C. 2007. Migrants, markets and multinationals: Competition among world cities for the highly skilled. GeoJournal 68:119.

Fairlie, Robert W. 2006. An extension of the Blinder-Oaxaca decomposition Technique to Logit and Probit Models. IZA Discussion Paper 1917. 
Fassmann, Heinz, Gerhard Hatz und Walter Matznetter. Hrsg. 2009. Wien - Städtebauliche Strukturen und gesellschaftliche Entwicklungen. Wien: Böhlau.

Foster, James E, und Michael C Wolfson. 2010. Polarization and the decline of the middle class: Canada and the US. Journal of economic inequality 8:247-273.

Franz, Yvonne. 2015. Gentrification in neighbourhood development. Case studies from New York City, Berlin and Vienna. Vienna: V\&R unipress.

Friedmann, John. 1986. The world city hypothesis. Development and change 17:69-83.

Fritsch, Nina-Sophie, Roland Teitzer und Roland Verwiebe. 2014. Arbeitsmarktflexibilisierung und wachsende Niedriglohnbeschäftigung in Österreich. Eine Analyse von Risikogruppen und zeitlichen Veränderungen. Österreichische Zeitschrift für Soziologie 39:91-110.

Fritsch, Nina-Sophie, Roland Verwiebe und Christina Liebhart. 2018. Arbeit und Berufe in Österreich. In Sozialer Wandel in Österreich 1986 bis 2016, Hrsg. Johann Bacher, Martina Beham-Rabanser, Alfred Grausgruber, Max Haller, Johanna Muckenhuber, Franz Höllinger und Roland Verwiebe. Wiesbaden: Springer.

Gächter, August. 2008. Migrationspolitik in Österreich seit 1945 [Migration policy in Austria since 1945]. Vienna: ZSI.

Le Galès, Patrick. 2002. European cities: Social conflicts and governance. Oxford: Oxford University Press.

Geißler, Rainer. 2014. Die Sozialstruktur Deutschlands. Wiesbaden: Springer VS.

Giesecke, Johannes, und Roland Verwiebe. 2010. Erwerbschancen und Arbeitsmarktintegration im wiedervereinigten Deutschland. In 20 Jahre Fall der Mauer - Bilanz der deutschen Einheit, Hrsg. Peter A Krause, und Ilona Ostner, 247-276. Frankfurt a. M.: Campus.

Goebel, Jan, Martin Gornig und Hartmut Häußermann. 2012. Bestimmt die wirtschaftliche Dynamik der Städte die Intensität der Einkommenspolarisierung? Resultate für deutsche Stadtregionen. Leviathan 40:371-395.

Görgl, Peter, Marco Helbich, Walter Matznetter und Heinz Fassmann. 2011. Spatial and social development trends of metropolitan Vienna: an Overview. In Urban Sprawl in Europe. Similarities or differences?, Hrsg. Viktória Szirmai, 107-140. Budapest: Aula Kiadó.

Grabka, Markus, und Joachim R. Frick. 2008. Schrumpfende Mittelschicht - Anzeichen einer dauerhaften Polarisierung der verfügbaren Einkommen? DIW-Wochenbericht 75:101-115.

Groh-Samberg, Olaf, Steffen Mau und Uwe Schimank. 2014. Lebensführung unter Druck? Die Statusarbeit der Mittelschichten. Weinheim: Beltz-Juventa.

Hamnett, Chris. 1994. Social polarisation in global cities: Theory and evidence. Urban Studies 31:401-424.

Hamnett, Chris. 2015. The changing occupational class composition of London. City 19:239-246.

Häußermann, Hartmut, und Walter Siebel. 1993. New York, Strukturen einer Metropole. Frankfurt a. M.: Suhrkamp.

Hermann, Christoph, und Jörg Flecker. 2009. Das Modell Österreich im Wandel. In Die Dynamik des „österreichischen Modells“. Brüche und Kontinuitäten im Beschäftigungs- und Sozialsystem, Hrsg. Hermann Christoph und Atzmüller Roland, 17-44. Berlin: edition sigma.

Herzog-Punzenberger, Barbara. 2017. Ungleichheit in der Einwanderungsgesellschaft: Intergenerationale Mobilität der angeworbenen Arbeitskräfte in Österreich: Springer.

Hoetker, Glenn. 2007. The use of logit and probit models in strategic management research: Critical issues. Strategic Management Journal 28:331-343.

Holm, Andrej. 2011. Gentrification in Berlin: neue Investitionsstrategien und lokale Konflikte. In Die Besonderheit des Städtischen. Entwicklungslinien der (Stadt)Soziologie, Hrsg. Heike Herrmann, Carsten Keller, Rainer Neef und Renate Ruhne, 213-232. Wiesbaden: VS Verlag für Sozialwissenschaften.

Huber, Florian J. 2013. Gentrifizierung in Wien, Chicago und Mexiko Stadt. Österreichische Zeitschrift für Soziologie 38:237-257.

Jann, Ben. 2006. Stata module to generate nonlinear decomposition of binary outcome differentials: Boston College Department of Economics: Statistical Software Components.

Kalter, Frank, und Nadia Granato. 2002. Demographic change, educational expansion and structural assimilation of immigrants. The case of Germany. ESR 18:199-216.

Kalter, Frank, und Nadia Granato. 2018. Migration und ethische Ungleichheit auf dem Arbeitsmarkt. In Arbeitsmarktsoziologie, Hrsg. Martin Abraham und Thomas Hinz. Wiesbaden: Springer VS.

Karlson, Kristian Bernt, Anders Holm und Richard Breen. 2012. Comparing regression coefficients between same-sample nested models using logit and probit: A new method. Sociological Methods \& Research 42:286-313.

King, Vera. 2009. Ungleiche Karrieren. Bildungsaufstieg und Adoleszenzverläufe bei jungen Männern und Frauen aus Migrantenfamilien. In Bildungsprozesse Jugendlicher und junger Erwachsener mit 
Migrationshintergrund, Hrsg. Vera King und Hans-Christoph Koller, 27-46. Wiesbaden: VS Verlag für Sozialwissenschaften.

Kogan, Irena. 2011. The price of being an outsider: Labour market flexibility and immigrants' employment paths in Germany. International Journal of Comparative Sociology 52:264-283.

Koser, Khalid, und John Salt. 1997. The geography of highly skilled international migration. International Journal of Population Geography 3:285-303.

Leschke, Janine, und Bela Galgögczi. 2015. Arbeitskräftemobilität in der EU im Abgesicht der Krise: Gewinner und Verlierer. WSI Mitteilungen.

Lohmann, Henning. 2010. Armut von Erwerbstätigen im europäischen Vergleich: Erwerbseinkommen und Umverteilung. Kölner Zeitschrift für Soziologie und Sozialpsychologie 62:1-30.

Lucifora, Claudio, Abigail McKnight und Wiemer Salverda. 2005. Low-wage employment in Europe: A review of the evidence. Socio Economic Review 3:259-292.

Massari, Riccardo, Maria Grazia Pittau und Roberto Zelli. 2009. A dwindling middle class? Italian evidence in the 2000s. Journal of Economic Inequality 7:333-350.

Mau, Steffen. 2012. Lebenschancen. Wohin driftet die Mittelschicht? Berlin: Suhrkamp.

May, Jon, Jane Wills, Kavita Datta, Yara Evans, Joanna Herbert und Cathy McIlwaine. 2007. Keeping London working: Global cities, the British state and London's new migrant division of labour. Transactions of the Institute of British Geographers 32:151-167.

McDowell, Linda, Adina Batnitzky und Sarah Dyer. 2009. Precarious work and economic migration: Emerging immigrant divisions of labour in Greater London's service sector. International Journal of Urban and Regional Research 33:3-25.

McKernan, Signe-Mary, und Caroline Ratcliffe. 2005. Events that trigger poverty entries and exits. Social Science Quarterly 86:1146-1169.

Mohr, Katrin. 2007. Soziale Exklusion im Wohlfahrtsstaat: Arbeitslosensicherung und Sozialhilfe in Großbritannien und Deutschland. Wiesbaden: VS Verlag für Sozialwissenschaften.

Musterd, Sako, und Wim Ostendorf. Hrsg. 2013. Urban segregation and the welfare state: Inequality and exclusion in western cities. London: Routledge.

Musterd, Sako, Szymon Marcińczak, Maarten van Ham und Tiit Tammaru. 2017. Socioeconomic segregation in European capital cities. Increasing separation between poor and rich. Urban Geography 28:1062-1083.

Nohl, Arnd-Michael. 2010. Von der Bildung zum kulturellen Kapital: Die Akkreditierung ausländischer Hochschulabschlüsse auf deutschen und kanadischen Arbeitsmärkten. In Kulturelles Kapital in der Migration, Hrsg. Arnd-Michael Nohl, Karin Schittenhelm, Oliver Schmidtke und Anja Weiß, 153-165. Wiesbaden: VS Verlag für Sozialwissenschaften.

OECD. 2008. The global competition for talent: Mobility of the highly skilled. Paris: OECD.

OECD. 2018. International migration outlook 2018. OECD Publishing. Paris: OECD.

Peixoto, Joao. 2001. The international mobility of highly skilled workers in transnational corporations. International Migration Review 35:1030-1053.

Piketty, Thomas. 2014. Capital in the twenty-first century. Cambridge: Belknap Press of Harvard University Press.

Reiner, Christian, Susanne Meyer und Sascha Sardadvar. 2017. Urban attraction policies for international academic talent: Munich and Vienna in comparison. Cities 61:27-35.

Riesenfelder, Andreas, Susanne Schelepa und Petra Wetzel. 2011. Beschäftigungssituation von Personen mit Migrationshintergrund in Wien. Endbericht. Wien: L\&R Sozialforschung.

Ryan, Louise, und Jon Mulholland. 2014. Trading places: French highly skilled migrants negotiating mobility and emplacement in London. Journal of Ethnic and Migration Studies 40:584-600.

Salt, John. 1992. Migration processes amongst the highly skilled in Europe. International Migration Review 26:484-505.

Sanderson, Matthew R, Ben Derudder, Michael Timberlake und Frank Witlox. 2015. Are world cities also world immigrant cities? An international, cross-city analysis of global centrality and immigration. International Journal of Comparative Sociology 56:173-197.

Sassen, Saskia. 1991. The global city. Princeton, NJ: Princeton University Press.

Sassen, Saskia. 2016. The global city: Enabling economic intermediation and bearing its costs. City \& Community 15:97-108.

Savage, Mike, Fiona Devine, Niall Cunningham, Mark Taylor, Yaojun Li, Johs Hjellbrekke, Brigitte Le Roux, Sam Friedman und Andrew Miles. 2013. A new model of social class? Findings from the BBC's Great British Class Survey experiment. Sociology 47:219-250.

Schiman, Stefan. 2018. Labor supply shocks and the beveridge curve: Empirical evidence from Austria. WIFO Working Papers, No. 568. 
Siegert, Manuel. 2013. Die Zufriedenheit der Migranten in Westdeutschland. Wiesbaden: Springer Fachmedien.

Sinning, Mathias, Markus Hahn und Thomas K Bauer. 2008. The Blinder-Oaxaca decomposition for nonlinear regression models. The Stata Journal 8:480-492.

Stadt Wien. 2014. 3. Wiener Integrations- \& Diversitätsmonitor 2011-2013. Wien: Stadt Wien, Magistratsabteilung 17 - Integration und Diversität.

Stadt Wien. 2016a. 4. Wiener Integrations- \& Diversitätsmonitor 2013-2016. Wien: Stadt Wien, Magistratsabteilung 17 - Integration und Diversität.

Stadt Wien. 2016b. Wien in Zahlen 2016. Wien: Magistratsabteilung 23.

Statistik Austria. 2014a. Verfügbares Haushaltseinkommen und äquivalisiertes Nettohaushaltseinkommen 2013. http://www.statistik.at/web_de/static/ergebnisse_im_ueberblick_verfuegbares_haushaltsein kommen_und_aequivalisier_022294.pdf (Zugegriffen: 22.10.2016).

Statistik Austria. 2015. Migration \& Integration: Zahlen, Daten, Indikatoren 2015. Vienna.

Tai, Po-Fen. 2006. Social Polarisation: Comparing Singapore, Hong Kong and Taipei. Urban Studies 43:1737-1756.

Till Tentschert, Ursula, Nadja Lamei und Martin Bauer. 2004. Armut und Armutsgefährdung in Österreich 2003: Ressortaktivitäten und Analysen. In Bericht über die soziale Lage 2003-2004, Hrsg. BMSG, 207-232. Wien: BMSG.

Trampusch, Christine. 2010. Employers, the state and the politics of institutional change: Vocational education and training in Austria, Germany and Switzerland. European Journal of Political Research 49:545-573.

Tucci, Ingrid, und Gerd G. Wagner. 2005. Einkommensarmut bei Zuwanderern überdurchschnittlich gestiegen. DIW-Wochenbericht 72:79-86.

Vandecasteele, Leen. 2011. Life course risks or cumulative disadvantage? The structuring effect of social stratification determinants and life course events on poverty transitions in Europe. ESR 27:246-263.

Verwiebe, Roland. 2010. Wachsende Armut in Deutschland und die These der Auflösung der Mittelschicht. Eine Analyse der deutschen und migrantischen Bevölkerung mit dem Sozio-ökonomischen Panel. In Dynamiken (in) der gesellschaftlichen Mitte, Hrsg. Peter A. Berger und Nicole Burzan, 159-179. Wiesbaden: VS Verlag für Sozialwissenschaften.

Verwiebe, Roland, Raimund Haindorfer, Lena Seewann, Cornelia Dlabaja und Sina Lipp. 2015. Zusammenleben in Wien. Einstellungen zu Zuwanderung und Integration. Werkstattbericht 152. Wien: Magistratsabteilung 18.

Verwiebe, Roland, Christoph Reinprecht, Raimund Haindorfer und Laura Wiesböck. 2017. How to succeed in a transnational labor market. job search and wages among Hungarian, Slovak and Czech commuters in Austria. International Migration Review 51:251-286.

Verwiebe, Roland, Lena Seewann, Margarita Wolf und Melek Hacioglu. 2016. ,I have to be very good in what I do'. Marginalisation and discrimination in the career-entry phase. Ex-periences and coping strategies among university graduates with a migrant background in Austria. JEMS 42:2468-2490.

Verwiebe, Roland, Tobias Troger, Laura Wiesböck, Roland Teitzer und Nina-Sophie Fritsch. 2014. Austria - The bastion of calm? Stability and change in inequalities in times of welfare state reforms and employment flexibilization. In Changing inequalities and societal impacts in rich countries: Thirty countries' experiences, Hrsg. Brian Nolan, Wiemer Salverda, Daniele Checchi, Ive Marx, Abigail Mcknight, István György Tóth und Herman G. Van De Werfhorst, 71-95. Oxford: Oxford University Press.

Watt, Paul. 2008. The only class in town? Gentrification and the middle-class colonization of the city and the urban imagination. International Journal of Urban and Regional Research 32:206-211.

Whelan, Christopher T., Brian Nolan und Bertrand Maitre. 2017. Polarization or „squeezed middle“ in the great recession?: A comparative European analysis of the distribution of economic stress. Social Indicator Research 133:163-184.

Wiedner, Jonas, und Johannes Giesecke. 2017. The dynamics of immigrant-native labor market differentials in West Germany, 1976-2012. RC28 Spring Meeting 2017. University of Cologne.

Wiesböck, Laura, Roland Verwiebe, Christoph Reinprecht und Raimund Haindorfer. 2016. The economic crisis as a driver of cross-border labour mobility? A multi-method perspective on the case of the central European region. Journal of Ethnic and Migration Studies:1-17.

Zimmermann, Klaus F. Hrsg. 2005. European migration: What do we know? Oxford: Oxford University Press. 
Bernhard Riederer 1981, Mag. Dr., wissenschaftlicher Mitarbeiter am Institut für Soziologie der Universität Wien und am Institut für Demographie der Österreichischen Akademie der Wissenschaften. Forschungsgebiete: Ungleichheit/Lebensqualität, Familie/Partnerschaft, regionale und internationale Vergleiche. Veröffentlichungen, u. a.: Elternschaft und Wohlbefinden: Kinder im individuellen, partnerschaftlichen und gesellschaftlichen Kontext. Wiesbaden 2018. Changes in the educational achievement of immigrant youth in Western societies: The contextual effects of national (educational) policies (mit R. Verwiebe). European Sociological Review 2015.

Lena Seewann 1989, MA, Universitätsassistentin am Institut für Soziologie, Universität Wien. Forschungsgebiete: Werte und Religion, Migration, Methoden der Sozialwissenschaften. Veröffentlichungen, u. a.: Der Umgang mit Ausgrenzungs- und Diskriminierungserfahrungen in der Berufseinstiegsphase. Das Beispiel von AkademikerInnen mit Migrationshintergrund in Österreich. Schweizer Zeitschrift für Soziologie 42; Lebensqualität in Wien im 21. Jahrhundert (mit R. Verwiebe, B. Riederer und T. Troger). Wien 2014.

Roland Verwiebe 1971, Univ.-Prof. Dr., Universitätsprofessor für Sozialstrukturforschung und quantitative Methoden, Institut für Soziologie, Universität Wien. Forschungsgebiete: Ungleichheit, Arbeitsmarkt, Migration. Veröffentlichungen, u. a.: The role of education for poverty risks revisited: Couples, employment and profits from work-family policies (mit T. Troger). Journal of European Social Policy 25, 2015; How to succeed in a transnational labor market. Job search and wages among Hungarian, Slovak and Czech commuters in Austria (mit C. Reinprecht, R. Haindorfer, L. Wiesböck). International Migration Review 51, 2017; „I have to be very good in what I do“. Marginalisation and discrimination in the career-entry phase. Experiences and coping strategies among university graduates with a migrant background (mit L. Seewann, M. Wolf, M. Hacioglu). Journal of Ethnic and Migration Studies 42, 2016. 